\title{
Monitoring von Freiflächeninanspruchnahme und -versiegelung für eine nachhaltige Raumentwicklung in Bayern
}

\author{
Constantin Meyer, Jan Christoph Peters, Michael Thiel, Joachim Rathmann, Hubert Job \\ Eingegangen: 23. Dezember 2020 - Angenommen: 11. März 2021 - Online veröffentlicht: 31. März 2021
}

\section{Zusammenfassung}

Im Freistaat Bayern wird intensiv diskutiert, wie die nach wie vor hohe Freiflächeninanspruchnahme für Siedlungs- und Verkehrszwecke reduziert werden kann. Wissenschaftliche Grundlage für Steuerungsansätze in der Stadt- und Regionalentwicklung sollte ein verbessertes staatliches Flächenmonitoring sein, welches über die amtliche Statistik und deren Hauptindikator "Siedlungs- und Verkehrsfläche“ hinaus auch die qualitative Dimension der Flächeninanspruchnahme einbezieht. Dafür stellt dieser Beitrag methodische

Constantin Meyer, Institut für Geographie und Geologie, Lehrstuhl für Geographie und Regionalforschung, Julius-Maximilians-Universität Würzburg, Am Hubland, 97074 Würzburg constantin.meyer@uni-wuerzburg.de

Jan Christoph Peters, Institut für Geographie und Geologie, Lehrstuhl für Geographie und Regionalforschung, Julius-Maximilians-Universität Würzburg, Am Hubland, 97074 Würzburg janchristophpeters@web.de

Dr. Michael Thiel, Institut für Geographie und Geologie, Lehrstuhl für Fernerkundung, Julius-Maximilians-Universität Würzburg, Oswald-Külpe-Weg 86, 97074 Würzburg

michael.thiel@uni-wuerzburg.de

Dr. Joachim Rathmann, Institut für Geographie und Geologie, Lehrstuhl für Geographie und Regionalforschung, Julius-Maximilians-Universität Würzburg, Am Hubland, 97074 Würzburg joachim.rathmann@uni-wuerzburg.de

Prof. Dr. Hubert Job, Institut für Geographie und Geologie, Lehrstuhl für Geographie und Regionalforschung, Julius-Maximilians-Universität Würzburg, Am Hubland, 97074 Würzburg hubert.job@uni-wuerzburg.de

(c) 2021 Meyer; licensee oekom verlag. This Open Access article is published under a Creative Commons Attribution 4.0 International License.
Erweiterungsansätze für das Flächenmonitoring vor, welche kleinräumige Analysen der Zersiedelung, Freiraumstruktur, Flächenversiegelung und Ökosystemleistungen am Beispiel des Landkreises Rhön-Grabfeld aufzeigen. Diese werden im Kontext der Debatte zu Ursachen und Steuerung der Freiflächeninanspruchnahme sowie zu aktuellen Anforderungen an das Flächenmonitoring diskutiert. Betont wird deren Bedeutung für das Monitoring rechtlicher Vorgaben und politischer Ziele zur nachhaltigen Flächennutzung.

Schlüsselwörter: Bayern - Flächenmonitoring Freiraumstruktur - Ökosystemleistungen - Siedlungs- und Verkehrsfläche $=$ Zersiedelung

\section{Monitoring of land consumption and soil sealing as a contribution to sustainable spatial development in Bavaria}

\begin{abstract}
In the federal state of Bavaria, there is currently intensive discussion on how land consumption for settlement and transport purposes can be reduced in the long term. In order to provide a solid scientific basis for steering instruments in urban and regional development, the official land use monitoring should be improved by including also the qualitative and structural dimension of land consumption in addition to existing official statistics and their main indicator 'settlement and traffic area'. For this purpose, the paper presents methodological extensions, which show small-scale analyses of urban sprawl, open space structure, soil sealing and ecosystem services using the example of the district of Rhön-Grabfeld. These methodological extensions are discussed in the context of the relevant debates on the causes and steering of land consumption as well as on current requirements for land use monitor-
\end{abstract}


ing, emphasising their importance for the monitoring of legal guidelines and political objectives on sustainable land use.

Keywords: Bavaria - Ecosystem services - Land use monitoring = Open spaces = Settlement and traffic area Urban sprawl

\section{Einführung}

Böden erfüllen zahlreiche Ökosystemleistungen (ÖSL): Neben der Filter-, Puffer- und Transformatorfunktion speichern sie Stoffe, beispielsweise Treibhausgase, und besitzen eine Habitatfunktion für zahlreiche (Mikro-)Organismen. Bodenüberbau und Versiegelungen haben vorwiegend negative Auswirkungen auf den Wasser- und Wärmehaushalt. Insbesondere bei einer dispersen Siedlungsentwicklung werden Lebensräume von Tieren und Pflanzen zerschnitten. Durch die Überbauung oder Versiegelung von Flächen werden demnach Ökosystemleistungen beeinträchtigt (Wessolek 2014: 18; Tobias/Conen/Duss et al. 2018: 2016; Rathmann/Dubrow/Eberhardt 2019: 17).

Die Freiflächeninanspruchnahme für Siedlungs- und Verkehrszwecke sowie Möglichkeiten zu deren Reduzierung werden derzeit sowohl in den Raumwissenschaften als auch in der Praxis von Stadt-, Regional- und Landesplanung intensiv diskutiert (Wiggering/Fischer/Penn-Bressel et al. 2009; ARL 2018; Behnisch/Kretschmer/Meinel 2018). In Deutschland bildet hierbei der Indikator der Veränderung der Siedlungs- und Verkehrsfläche (SuV) aus der amtlichen Flächenstatistik die zentrale Grundlage zur Beurteilung von quantitativen Trends der Siedlungsentwicklung. Auf diesen Indikator bezogen existieren politische Zielsetzungen wie das 30-ha-Ziel auf Bundesebene (Bundesregierung 2002: 189), welches ursprünglich für das Jahr 2020 anvisiert wurde. In einer Neuauflage der Nachhaltigkeitsstrategie der Bundesregierung wurde dieses Ziel als ,30ha-minus-X“ Zielsetzung (Bundesregierung 2016: 159) auf das Jahr 2030 verschoben.

Auch die Bayerische Staatsregierung hat im Zuge der aktuellen „Flächensparoffensive“ eine ,5-ha-Richtgröße“, welche den bayerischen Anteil an den bundesweiten 30 ha/Tag repräsentiert, forciert. Diese wurde in der jüngsten Novellierung in das Bayerische Landesplanungsgesetz (Art. 6 Abs. 2 Nr. 3 S. 6 BayLplG) ${ }^{1}$ aufgenommen - allerdings nur als unverbindliche Richtgröße, welche spätestens bis zum Jahr 2030 erreicht werden soll. Das stellt eine lan-

\footnotetext{
1 Bayerisches Landesplanungsgesetz (BayLplG) vom 25. Juni 2012, das zuletzt durch das Gesetz vom 23. Dezember 2020 geändert worden ist.
}

desentwicklungspolitische Volte dar, denn zuvor wurden mit den 2013 und 2018 vorgenommenen Novellierungen des Bayerischen Landesentwicklungsprogrammes raumplanerische Ziele aufgeweicht und somit Prozesse der Zersiedelung nicht ausreichend vermieden. Allen voran die Wirkung der sogenannten Anbindegebot-Norm² ist durch zahlreiche Ausnahmetatbestände stark geschwächt worden (Job/ Mayer/Kraus 2014: 344; Job/Weidlich/Meyer 2019: 43).

Als Freiflächeninanspruchnahme wird der Zuwachs an Siedlungs- und Verkehrsfläche verstanden. Dieser Definition folgend werden in Deutschland täglich rund 56 ha Freifläche in Siedlungs- und Verkehrsfläche umgewandelt (Statistisches Bundesamt 2020: o. S.). In Bayern beträgt dieser Wert ungefähr 11,1 ha (eigene Berechnungen auf der Grundlage von LfStat 2020a). ${ }^{3}$ Unbebauter Boden als Freiraum ist ein wichtiges, nicht vermehrbares Gut - gerade in Zeiten, in denen die Knappheit bezahlbaren Wohnraums stark kritisiert und die Ausweisung von mehr Bauland als pauschale Lösung propagiert wird. Deshalb tun räumlich differenzierte und planerisch fundierte Lösungsansätze für die konsequente Umsetzung eines raumordnerischen Flächensparziels Not.

Das flächengrößte Bundesland Deutschlands dient als Fallbeispiel, weil hier die Freiflächeninanspruchnahme seit Jahren relativ hoch ist und eine gesellschaftliche Sensibilisierung zum Thema „Flächensparen“ existiert. Das hat die breite Unterstützung des 2018 beantragten Volksbegehrens „Betonflut eindämmen. Damit Bayern Heimat bleibt“ gezeigt. $^{4}$

Vor der Implementierung planerischer Lösungsansätze sollte eine fundierte Analyse und Neubewertung der Freiflächenneuinanspruchnahme erfolgen. Hierzu wird nachfolgend ein konzeptioneller Vorschlag samt beispielhafter Anwendung für ein nachvollziehbares Flächenmonitoring vorgestellt, welcher zusätzlich zur Betrachtung der Siedlungsund Verkehrsfläche vorsieht, mittels der Indikatoren „Gewichtete Zersiedelung“ und „Freiraumstruktur“ (vgl. Peters 2020), einer Analyse der tatsächlichen Bodenversiegelung sowie der durch Landnutzungsänderungen induzierten Veränderungen von Ökosystemleistungen die Entwick-

\footnotetext{
2 Das sogenannte Anbindegebot zur Vermeidung von Zersiedelung ist in Kapitel 3.3 des Bayerischen Landesentwicklungsprogramms (LEP) verankert und besagt, dass neue Siedlungsflächen möglichst in Anbindung an geeignete (bestehende) Siedlungseinheiten auszuweisen sind.

3 Darstellung des gleitenden Vierjahresmittels für 2018, um beide Werte (Bayern und Deutschland gesamt) methodisch vergleichen zu können.

${ }^{4}$ https://www.gruene-fraktion-bayern.de/themen/ landesentwicklung-und-tourismus/2019/

betonflut-eindaemmen-damit-bayern-heimat-bleibt/ (08.02.2021).
} 
lung besser zu erfassen. Der in diesem Beitrag vorgestellte Ansatz weist grundsätzlich Ähnlichkeiten zu einer Reihe von bestehenden Indikatorensystemen auf, auch wenn diese durch unterschiedlichen Anspruch, Umfang und Schwerpunkt gekennzeichnet sind (vgl. z. B. LUBW 2007; Siedentop/Heiland/Lehmann et al. 2007; Penn-Bressel 2009; Klein/Wettemann/Esch et al. 2010; Goetzke 2018). Als Zielsetzung des Ansatzes steht allerdings nicht, wie in den meisten genannten Publikationen, die umfassende statistische Bewertung der Flächen- und Siedlungsentwicklung in administrativen Einheiten auf Basis der amtlichen (Flächen-)Statistik im Fokus. Vielmehr werden ausgewählte methodische Erweiterungen vorgestellt, welche zwei zentrale Vorteile aufweisen: Zum einen werden durch Rasterauflösungen kleinräumige Differenzierungen der Flächeninanspruchnahme und Freiraumstruktur ermöglicht, die raumzeitliche Veränderungen präziser erfassen als Vergleiche auf Landkreisoder Gemeindeebene. Zum anderen bietet der Ansatz bei einer vergleichsweise geringen Anzahl an Indikatoren eine relativ umfassende Berücksichtigung der Thematik durch die gleichrangige Einbeziehung von Quantität (SuV-Entwicklung), Qualität (Bodenversiegelung und Ökosystemleistungen) und Struktur (Zersiedelung und Freiraumstruktur) der Freiflächeninanspruchnahme. Dadurch wird die Anforderung berücksichtigt, Konfiguration und Komposition der Flächennutzung in die Raumbeobachtung zu integrieren (Fina 2018: 119), um Entscheidungsgrundlagen für Planungsakteure zu verbessern.

Der vorliegende Beitrag ist in sieben Kapitel gegliedert. Während in den Kapiteln 2 und 3 die Rahmenbedingungen und Treiber der Flächeninanspruchnahme sowie die Anforderungen an das Flächenmonitoring skizziert werden, führt Kapitel 4 kurz in das bayerische Untersuchungsgebiet (Landkreis Rhön-Grabfeld) ein. Aufbauend auf einem Kapitel zur Datengrundlage des Artikels (Kapitel 5), werden die vorgeschlagenen methodischen Erweiterungen in Kapitel 6 aufgezeigt und erläutert, bevor in Kapitel 7 eine abschlieBende Diskussion der Relevanz stattfindet.

\section{Treiber der Flächeninanspruch- nahme und planerisch-politische Rahmensetzungen}

Miosga (2019: $8 \mathrm{f}$.) hat für Bayern herausgearbeitet, dass es vor allem der Wohnungsneubau und weniger die Gewerbegebiete sind, die Zersiedelung und Flächeninanspruchnahme verursachen. Demnach wachsen besonders die kleinen Gemeinden unter 6.500 Einwohner/-innen stark in die Umgebung. Warum ist das so und wie gestalten sich die Rahmendaten außerhalb der juridischen Setzungen der Raumplanung?
Flächendeckend hat die mittlere Pro-Kopf-Wohnfläche in Bayern von rund $19 \mathrm{~m}^{2}$ im Jahr 1960 auf rund $48 \mathrm{~m}^{2}$ in 2017 zugenommen (StMWi 2019: 19), das heißt, die Konsumansprüche an das Wohnen nahmen zu. Der Zuwachs liegt unter anderem daran, dass der Pro-Kopf-Wohnflächengebrauch mit dem Alter kontinuierlich ansteigt. Hierfür verantwortlich zeigen sich vor allem steigende Einkommen sowie die unter dem Stichwort Remanenzeffekt bekannten Umzugsstarrheiten bei einer Verkleinerung der Haushaltsgröße von Familien (Henger/Voigtländer 2019: 5). Auch die Zahl der Einpersonenhaushalte wächst kontinuierlich (StMWi 2019: 19). Folglich ist der Bedarf an Wohnungen vielerorts deutlich angestiegen. Hinzu kommt die dauerhafte Niedrigzinspolitik, die viele Anleger/-innen in Immobilien hat investieren lassen, sowie die Binnenwanderung, bei der sich die Zuzüge der insbesondere jungen Bevölkerung auf Verdichtungsräume konzentriert (Bräuninger/Schnaars 2017: 228).

Die demographischen Trends führen zu zunehmenden Stadt-Land-Disparitäten. Besonders Entwicklungen in ländlich-peripheren Regionen, welche trotz demographischer Schrumpfung oder Stagnation durch ein Wachstum von Siedlungs- und Verkehrsflächen geprägt sind, verdeutlichen jedoch, dass auch der Bedeutung angebotsorientierter kommunaler Baulandpolitik Rechnung getragen werden muss (Siedentop/Junesch/Straßer et al. 2009: 103). Während in einigen Großstädten enorme Wohnungsknappheit herrscht, sind viele strukturschwache, ländlich geprägte Regionen mit einem Überangebot von Wohnraum mit hohen Leerständen gekennzeichnet (Baldenius/Kohl/Schularick 2019: 34; Henger/Voigtländer 2019: 22). In 31 von 401 deutschen Kreisen liegt die Quote (Verhältnis von Baufertigstellungen und Baubedarf) dessen, was zwischen 2016 und $2018 \mathrm{mehr}$ gebaut wurde, als der durch die Entwicklung der Bevölkerung prognostizierte Wohnungsbedarf angibt, bei über $200 \%$. Vier peripher gelegene bayerische Kreise gehören derzeit zu den Spitzenreitern der Wohnungsbauüberhänge in Deutschland, darunter auch Rhön-Grabfeld (Henger/ Voigtländer 2019: 28 ff.).

Die Möglichkeiten, Freiflächeninanspruchnahme differenzierter zu betrachten, ergeben sich nicht nur aus den zunehmenden Möglichkeiten räumlicher Analysetechniken mittels Geographischer Informationssysteme (GIS). Auch betreffend den für die Stadt- und Raumentwicklung relevanten Rechtsgrundlagen besteht Handlungsbedarf zur Entwicklung, Sicherung und Wiederherstellung bedeutsamer ökologischer Flächenfunktionen (vgl. z. B. § 1a Abs. 2 
BauGB $^{5}$, § 2 Abs. 2 Nr. 6 ROG $^{6}$ oder Art. 6 Abs. 2 Nr. 8 BayLplG). Eine verringerte Freiflächeninanspruchnahme muss mit der Einführung eines differenzierten Flächenmonitorings verbunden sein. Hierbei sollte unbedingt die Erfassung der Flächenversiegelung einbezogen werden, um möglichst kleinräumig Handlungserfordernisse erkennen und eine effektive planerische Steuerung anstreben zu können. Auch in der Förderpolitik des Freistaates Bayern wurde 2018 begonnen, Flächenentsiegelungen als Handlungsfeld zu entwickeln. Neben der verwandten Förderinitiative „Innen statt außen“ sowie dem Förderprogramm „Erfassung der Innenentwicklungspotentiale" besteht seitdem die neue Förderinitiative „Flächenentsiegelung“ der Städtebauförderung in Bayern (StMB 2018: 8).

\section{Anforderungen an das Flächenmonitoring}

Flächenmonitoring nimmt eine Unterstützungsfunktion für Entscheidungen in der räumlichen Planung ein. Es umfasst die drei Bestandteile Beobachtung (laufende Erfassung), Überwachung (Beobachtung bestimmter Objekte) und Kontrolle (Delta Soll-/Ist-Zustand) (Jacoby 2009: 11). Die rechtlichen Vorgaben zum staatlichen Flächenmonitoring sind jedoch kaum konkretisiert: Das Bayerische Landesplanungsgesetz (BayLplG) etwa verpflichtet die Landesplanungsbehörden in Artikel 31 nur allgemein, raumbedeutsame Tatbestände und Entwicklungen fortlaufend zu erfassen, zu verwerten und zu überwachen.

Das Monitoring der Entwicklung der Flächennutzung beruht in Deutschland maßgeblich auf den Daten zur „Flächenerhebung nach Art der tatsächlichen Nutzung“, welche durch die Umstellung vom Automatisierten Liegenschaftsbuch (ALB) auf das GIS-basierte Amtliche Liegenschaftskatasterinformationssystem (ALKIS) in jüngerer Vergangenheit methodische Brüche erfahren hat (Beckmann/Dosch 2018a: 7).

Der derzeit genutzte Nachhaltigkeitsindikator „Anstieg der Siedlungs- und Verkehrsfläche (in ha/Tag)“" ist vergleichsweise transparent und einfach nachzuvollziehen. Als Berechnungsgrundlage setzt sich die Siedlungs- und Verkehrsfläche aus Gebäude- und Freiflächen, Betriebsflächen (ohne Abbauland), Erholungsflächen und Verkehrsflächen sowie Friedhöfen zusammen (Beckmann/Dosch 2018a: 7).

\footnotetext{
${ }^{5}$ Baugesetzbuch in der Fassung der Bekanntmachung vom 3. November 2017, das zuletzt durch Artikel 2 des Gesetzes vom 8. August 2020 geändert worden ist.

6 Raumordnungsgesetz vom 22. Dezember 2008, das zuletzt durch Artikel 5 des Gesetzes vom 3. Dezember 2020 geändert worden ist.
}

Diese Daten bringen für das amtliche Flächenmonitoring mehrere Probleme mit sich: Zum einen macht die Größe der Siedlungs- und Verkehrsfläche keine Aussage über den Grad der Versiegelung, da beispielsweise sowohl Hausgärten als auch Verkehrsbegleitgrün nicht gesondert betrachtet werden. Zum anderen ist ein rein quantitatives Verständnis von Flächeninanspruchnahme nicht weit genug gefasst, vernachlässigt es doch die qualitative Dimension der Zersiedelung (Schwarzak/Behnisch 2017: 79 ff.). Im Wesentlichen dreht sich die Kritik an dieser Art der Erfassung um den fehlenden Einbezug der Qualität unterschiedlicher Flächennutzungen. Veränderungen der Siedlungs- und Verkehrsfläche sagen nicht notwendigerweise etwas über den Verlust an ökologischer Qualität oder landwirtschaftlicher Bonität von Flächen bzw. die Folgekosten, z. B. hinsichtlich infrastruktureller Ver- und Entsorgung, aus.

Eine verstärkte qualitative Betrachtung scheint demzufolge sinnvoll, denn die negativen Folgen der Zersiedlung sind unter anderem steigende Kosten für öffentliche und private Haushalte (z. B. für öffentliche Infrastrukturen) (Schiller/Siedentop 2005: 83f.), Einwirkungen auf die Umwelt (Biodiversitätsverlust) sowie eine Zunahme des Verkehrs (Emissionen) (Jaeger/Schwick/Hennig et al. 2015: $18 \mathrm{f}$.). Vor dem Hintergrund des Bestrebens der Bundesregierung in der Neuauflage der Nachhaltigkeitsstrategie (Bundesregierung 2016: 38), neben der Begrenzung des SuV-Wachstums auch den einwohnerbezogenen Freiraumverlust zu verringern sowie die Siedlungsdichte konstant zu halten, fordern Beckmann und Dosch (2018a: 22; 2018b: 19) ein erweitertes Flächenmonitoring, das ergänzende Bausteine wie die Einbeziehung der Indikatoren Bodenversiegelung, Wohnflächen- und Siedlungsdichte, Innenentwicklungspotenziale oder auch Zersiedelung enthalten soll.

Methodisch ist darauf hinzuweisen, dass das der Flächenstatistik zugrunde liegende ALKIS-System nach wie vor einem ständigen Modellwandel unterliegt und daher besonders im bundesweiten Vergleich nur bedingt für ein kontinuierliches Flächenmonitoring geeignet ist, auch aufgrund zeitlicher Verzögerungen bei der Aufnahme von Flächennutzungsänderungen (Meinel/Henger/Krüger et al. 2020: 236). Das räumlich geringer aufgelöste Amtliche Topographisch-Kartographische Informationssystem (ATKIS), welches etwa auch der Plattform „IÖR-Monitor“ zugrunde liegt und als (Geo-)Datengrundlage für die in diesem Beitrag vorgestellten Ansätze zur Gewichteten Zersiedelung und Freiraumstruktur dient, weist hingegen robustere und thematisch höher aufgelöste Informationen auf (Meinel 2020: 109). 


\section{Untersuchungsgebiet}

Als Untersuchungsgebiet für die beispielhafte Anwendung der dargelegten methodischen Erweiterungen wurde der nordbayerische Landkreis Rhön-Grabfeld gewählt. Denn hier weisen die Siedlungsdichte mit 791,5 Einwohnern pro $\mathrm{km}^{2}$ Siedlungs- und Verkehrsfläche sowie die EinwohnerArbeitsplatz-Dichte mit 108,7 pro $\mathrm{km}^{2}$ Werte auf, die auf Kreisebene zu den niedrigsten in ganz Bayern zählen. ${ }^{7}$ Zudem zeigt eine Untersuchung des Bayerischen Landesamts für Umwelt (LfU 2017: 70), dass Rhön-Grabfeld mit durchschnittlich $682 \mathrm{~m}^{2}$ versiegelter Fläche pro Einwohner an dritter Stelle beim Vergleich der 96 bayerischen Kreise steht (vgl. Job/Engelbauer/Engels 2019: 70). Hinzu kommt der mit rund $400 \%$ deutschlandweit höchste Wert der Wohnungsbauüberhänge auf Kreisebene (Henger/Voigtländer 2019: 36).

Gleichzeitig besitzt der Landkreis Rhön-Grabfeld ein hohes naturräumliches Potenzial. Er ist Teil des länderübergreifenden UNESCO-Biosphärenreservats Rhön ${ }^{8}$, wobei bedeutsame Lebensräume an vielen Stellen durch verschiedene, zum Teil sehr große und naturschutzfachlich hochwertige, besonders von extensiver Grünlandwirtschaft abhängige Natur- und Landschaftsschutzgebiete bzw. FFH-Gebiete $^{9}$ geschützt sind. Eine vereinfachte Darstellung von Ökosystemleistungen erfolgt hier für Flächen des Bayerischen Vertragsnaturschutzprogrammes (VNP). Dieses dient dazu, die Bayerische Biodiversitätsstrategie umzusetzen und bezogen auf vier Biotoptypen (Acker, Wiese, Weide, Teich) eine extensive Bewirtschaftung wertvoller Lebensräume zu fördern. ${ }^{10}$

Naturräumlich lässt sich der Landkreis grob in die Landschaftsräume des Mittelgebirges Rhön, das Grabfeld und Henneberger Hügelland sowie die Haßberge einteilen. Der nördlichste Landkreis Bayerns bildet teilweise die Landesgrenze zu Thüringen im Nordosten sowie zu Hessen im Nordwesten. Der peripheren Lage im strukturschwachen ländlichen Raum geschuldet, verliert der Kreis im Rahmen des demographischen Wandels an Bevölkerung, verbunden mit einer Verschiebung der Altersstruktur (LfStat 2020b: 7).

\footnotetext{
7 https://www.inkar.de (08.02.2021).

8 https://www.biosphaerenreservat-rhoen.de/ (03.03.2021).

9 http://www.ffh-gebiete.de/ (03.03.2021).

10 https://www.stmuv.bayern.de/themen/naturschutz/ naturschutzfoerderung/vertragsnaturschutzprogramm/ index.htm (09.02.2021).
}

\section{Datengrundlagen}

Zur Berechnung des Indikators „Gewichtete Zersiedelung“ wurde das ATKIS Basis-DLM verwendet, das vom Bayerischen Landesamt für Digitalisierung, Breitband und Vermessung zur Verfügung gestellt wurde. Die Einwohnerdichte wurde auf der Grundlage der Zensus-2011-Daten im 100-m-Gitter der Statistischen Ämter des Bundes und der Länder ermittelt, da diese die genaueste räumliche Auflösung von Bevölkerungsdaten bieten. Um eine unterschiedliche Entwicklung der Bevölkerung zwischen Gemeinden seit 2011 abzubilden, wurden die Daten des Zensus 2011 anhand der Bevölkerungsfortschreibung zum Stichtag 31.12.2019 mit einem Korrekturfaktor fortgeschrieben. Als Datengrundlage für Arbeitsplätze wurde die Statistik zu den sozialversichungspflichtig Beschäftigten vom Bayerischen Landesamt für Statistik gewählt. Da diese nur auf Gemeindeebene vorliegt, wurden die Daten auf Flächen, in denen potenziell Arbeitsplätze vorhanden sein könnten, anhand entsprechender ATKIS-Objektarten disaggregiert. ${ }^{11}$ Die Untersuchungseinheit, ein 500-m-Gitter, stammt vom Geodatenzentrum des Bundesamtes für Kartographie und Geodäsie (BKG). Zur Berechnung des Indikators „Freiraumstruktur" wurde ebenfalls das ATKIS Basis-DLM zugrunde gelegt. Die Untersuchungseinheit ist das gleiche 500-m-Gitter des BKG, wie es für die Darstellung der „Gewichteten Zersiedelung“ verwendet wurde.

Die Modellierung der Bodenversiegelung baut auf zwei frei verfügbaren Datensätzen auf: dem Versiegelungsdatensatz des Copernicus Land Monitoring Service ${ }^{12}$ für das Jahr 2015 (Langanke 2016: 12-17) sowie Daten zu den Straßeninformationen aus OpenStreetMap (OSM) ${ }^{13}$ Der Versiegelungsdatensatz hält flächendeckend Versiegelungsinformationen der Siedlungsräume im Rasterformat mit einer räumlichen Auflösung von $20 \mathrm{~m}$ vor. Der Vektordatensatz der Straßen aus OSM wurde unter Verwendung des Softwarepaketes R unter Einbindung der Bibliothek „osmar“ eingelesen, um ihn mit der Versiegelungsinformation zu verschneiden.

Zur Monetarisierung von Ökosystemleistungen wird am Beispiel der Leistung „Klimaschutz“ die Veränderung der Kohlenstoffspeicherung durch eine landwirtschaftliche Nut-

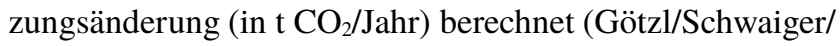

\footnotetext{
11 Mit der Durchführung des Zensus 2021 wird in naher Zukunft ein aktuellerer Datensatz zur Verfügung stehen. Insbesondere hinsichtlich der verfügbaren Daten zu Arbeitsplätzen bedarf es Nachbesserungen. Diese sind lediglich auf Gemeindeebene vorhanden und beachten derzeit keine selbstständigen Erwerbstätigen.

12 https:// land.copernicus.eu/ (09.02.2021).

13 https://openstreetmap.org (09.02.2021).
} 
Sonderegger et al. 2011: 31; Staub/Ott/Heusi et al. 2011: 46; Albert/Burkhard/Daube et al. 2015: 18); dazu muss der organische Kohlenstoff im Boden quantifiziert werden. Dies erfolgt unter der Annahme einer Umwandlung von Grünland in Ackerflächen, basierend auf Daten für Referenzbodeneinheiten zum organischen Kohlenstoff aus der Bodenübersichtskarte (BÜK25) des Landkreises Rhön-Grabfeld. Die Bodenübersichtskarte liegt im Maßstab 1:25.000 für Bayern vor und enthält Referenzbodeneinheiten, zu denen Bodendaten vorhanden sind. Diese beinhaltet Bodenschätzungsdaten sowie die geologische Karte im Maßstab 1:25.000. Zusätzlich werden die Beschreibung und die Abgrenzung der Bodeneinheiten in jedem Kartenblatt mit 300 bis 800 Bohrungen unterstützt. In einem weiteren Schritt ist der Kohlenstoffgehalt für Grün- und Ackerland zu berechnen, was auf Angaben von Poeplau, Don, Vesterdal et al. (2011: 2421) zur durchschnittlichen Kohlenstoffabnahme bei der Umwandlung von Grün- in Ackerland basiert.

\section{Methodische Ansätze und beispielhafte Anwendung}

\subsection{Gewichtete Zersiedelung und Freiraumstruktur}

Schwick, Jaeger und Kienast (2011: 3) beschreiben Zersiedelung als ein optisch wahrnehmbares Phänomen, das umso stärker ist, je mehr Fläche bebaut ist, je weiter diese Siedlungsfläche gestreut und je geringer die Ausnutzung durch Wohn- und Arbeitsplätze ist. Der Zersiedelungsgrad als Ergebnis des Indikators „Gewichtete Zersiedelung“ wird erklärt als „das Ausmass der Bebauung der Landschaft mit Gebäuden und ihrer Streuung, im Verhältnis zur Ausnützung der überbauten Flächen für Wohn- und Arbeitszwecke“ (Schwick/Jaeger 2010: 10). Die „Gewichtete Zersiedelung" nach Jaeger/Schwick (2014) bietet die Möglichkeit, zum einen eine Landschaftsbewertung anhand eines Multiindikators vorzunehmen und zum anderen nachfolgende drei Teilindikatoren zu differenzieren.

Die Dispersion ergibt sich aus mittleren gewichteten Abständen zwischen Siedlungspunkten, wobei die Gewichtung in unmittelbarer Nachbarschaft von Siedlungspunkten stark ansteigt und danach konstant bleibt. Die Dispersion ist besonders hoch, wenn die Siedlungspunkte weit gestreut liegen. Die Berechnung erfolgt innerhalb eines Beobachtungshorizontes, der auf 2 Kilometer festgesetzt ist (Jaeger/Schwick 2014: 12). Die urbane Durchdringung setzt die Siedlungsfläche in ein Verhältnis zur Gesamtfläche des Untersuchungsgebiets unter Berücksichtigung der Dispersion und ermöglicht somit die Vergleichbarkeit verschiedener Teilräume (Jaeger/Schwick 2014: $10 \mathrm{f}$.).
In diesem Teilindikator spiegelt sich auch die herkömmliche Erfassung der Siedlungs- und Verkehrsfläche in Form des Anteils der Siedlungsfläche an der Gesamtfläche des Untersuchungsraumes wider. Der Ausnutzungsdichte liegt die Annahme zugrunde, dass die Ausnutzung einer Fläche höher ist, je höher die Summe aus Arbeitsplätzen und Einwohnern in der Untersuchungseinheit ist (Schwarzak/Behnisch 2017: 83). Beim Zusammenfügen der einzelnen Indikatoren in den Multiindikator werden sowohl Dispersion als auch Ausnutzungsdichte gewichtet, um die Unterschiede innerhalb der Teilindikatoren deutlicher herauszustellen. Flächen mit starker Streuung und geringer Einwohner- und Arbeitsplatzdichte werden so deutlicher dokumentiert (Jaeger/Schwick 2014: 9 ff.). Die Vorteile des Indikators liegen somit in den trennscharfen, zugrunde liegenden Definitionen, methodische Restriktionen der „Gewichteten Zersiedelung“ bestehen vor allem in der Datenverfügbarkeit (vgl. Kapitel 5).

Um die Unterschiede zwischen „Gewichteter Zersiedelung“" und der bisherigen Erfassung von Flächeninanspruchnahme durch den „Anteil der Siedlungs- und Verkehrsfläche an der Gesamtfläche“ zu eruieren, wurde Letztere für den Landkreis Rhön-Grabfeld in einem 500-m-Raster dargestellt (vgl. Abbildung 1). Bad Neustadt an der Saale als Kreisstadt sticht hervor, deutlich setzen sich auch die Mittelzentren Bad Königshofen im Grabfeld und Mellrichstadt von den restlichen Gemeinden ab. Die „Gewichtete Zersiedelung" wurde ebenfalls anhand eines 500-m-Rasters berechnet. Die Daten wurden entsprechend der Vorschläge von Schwarzak und Behnisch (2017: 86) klassifiziert, wobei $<0,5$ Durchsiedelungseinheiten pro $\mathrm{m}^{2}\left(\mathrm{DSE} / \mathrm{m}^{2}\right)$ nahezu keine Zersiedlung darstellt und $>16 \mathrm{DSE} / \mathrm{m}^{2} \mathrm{sehr}$ starke Zersiedlung (vgl. Abbildung 2). Klar zu erkennen sind die größeren Gemeinden des Landkreises, angefangen von Bischofsheim in der Rhön und Bad Neustadt an der Saale, bis hin zu Mellrichstadt sowie Bad Königshofen im Grabfeld. Darüber hinaus sind Siedlungsmuster entlang der Bundesstraßen zu sehen. Diese sind von West nach Ost die B 279 (Bischofsheim in der Rhön bis Bad Königshofen im Grabfeld) sowie von Nord nach Nordwest die B 285 (Mellrichstadt bis Nordheim vor der Rhön). Parallel zur A 71 in Nord-Süd Richtung finden sich keine Siedlungen, lediglich parallel dazu auf der Strecke Mellrichstadt - Bad Neustadt an der Saale. Insbesondere Bad Neustadt an der Saale fällt durch hohe Zersiedlungswerte auf, was auf die Funktion als Oberzentrum zurückzuführen ist.

Die aggregierte Zersiedelung für den Landkreis beträgt 2,13 DSE $/ \mathrm{m}^{2}$, was einer leichten bis mittleren Zersiedlung gleichkommt. Der bayerische Durchschnitt liegt bei 3,65 

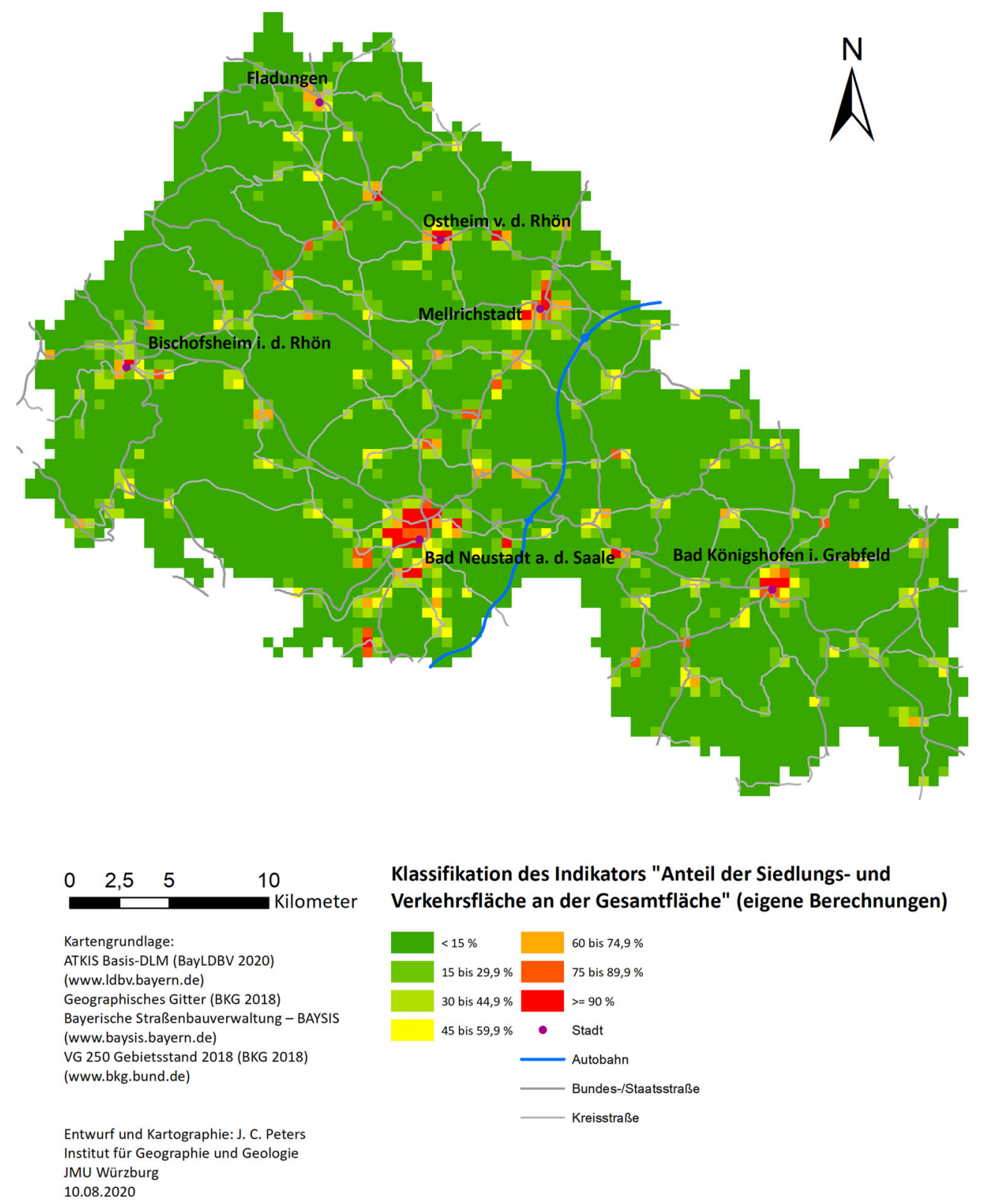

Klassifikation des Indikators "Anteil der Siedlungs- und Verkehrsfläche an der Gesamtfläche" (eigene Berechnungen)

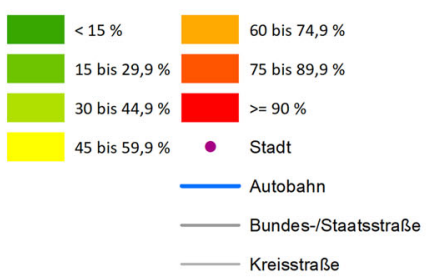

Abbildung 1 Anteil der Siedlungs- und Verkehrsfläche an der Gesamtfläche (500-m-Raster) im Landkreis Rhön-Grabfeld

DSE $/ \mathrm{m}^{2} .{ }^{14}$ Auf der Karte (vgl. Abbildung 2) sind schwach bis nicht zersiedelte Räume an den großen, grün eingefärbten Flächen zu erkennen. Hierbei handelt es sich unter anderem um Holzbodenfläche und Schutzgebiete. Ebenso zeigt die "Gewichtete Zersiedelung“ im Gegensatz zum SuVAnteil deutlicher die Ausbreitung von Siedlungsflächen, da über den Teilindikator Dispersion auch Flächen im Rand-

${ }_{14}$ Vgl. IÖR-Monitor: https://monitor.ioer.de/?rid=3536 (09.02.2021), Stand der Daten: 2010. bereich von Siedlungen hervorgehoben werden. In solchen Räumen treten höhere Siedlungsfolgekosten auf.

Komplementär zum methodischen Ansatz der „Gewichteten Zersiedelung“, welcher sich auf besiedelte Flächen bezieht, wird ein räumlicher (Multi-)Indikator zur Bewertung der Freiraumstruktur (Peters 2020) angewandt. Für diesen Ansatz wird Freiraum als unbebaute und unzerschnittene Landschaft definiert. Zur Untersuchung des Freiraums werden diskrete Landschaftselemente, bei denen es sich um die kleinste als weitgehend homogen anzusehende Nutzungseinheit handelt, betrachtet. Die Freiraumstruktur ergibt sich aus 


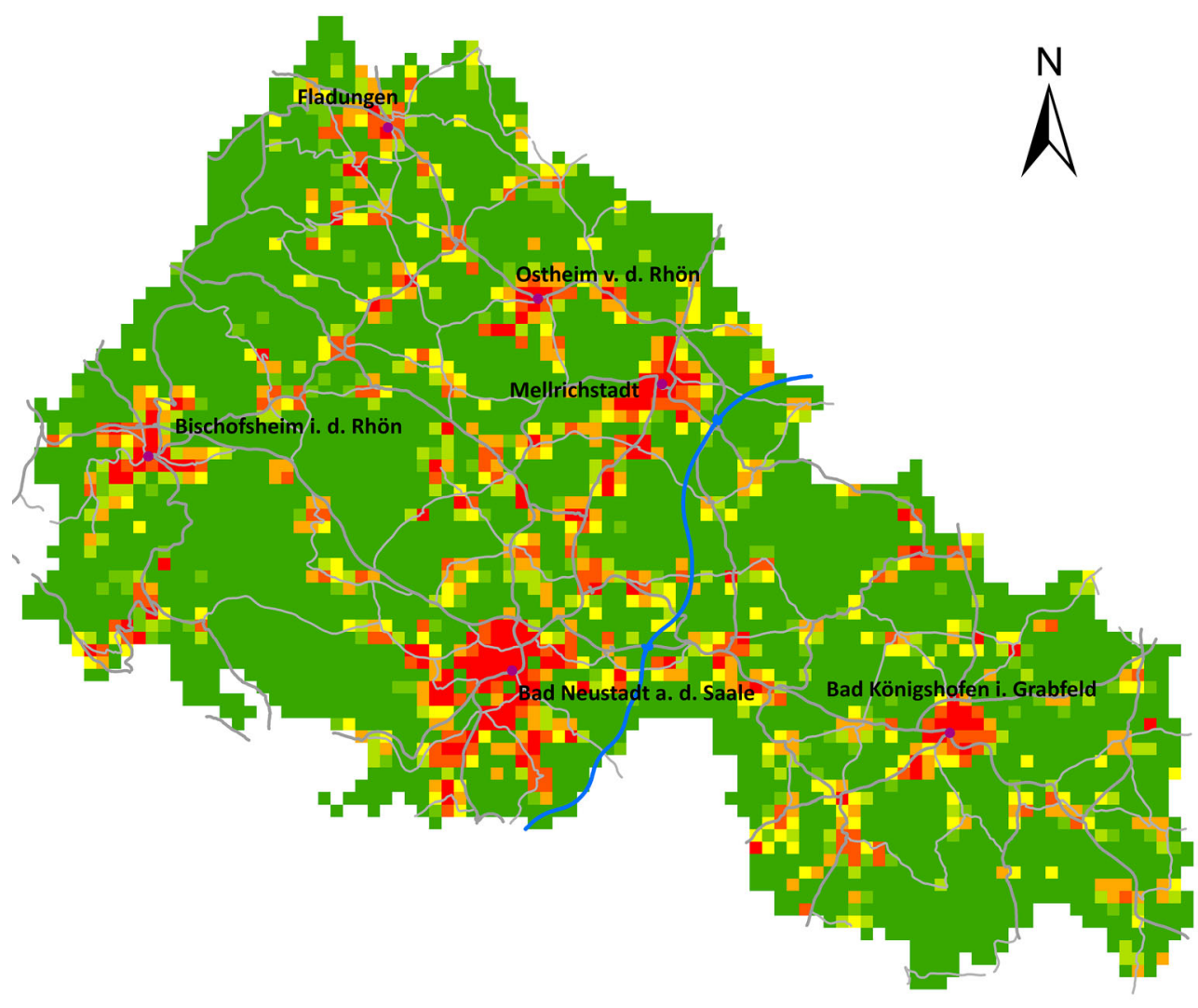

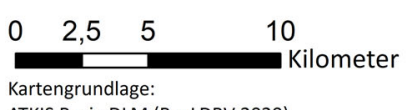

\section{Klassifikation des Indikators "Gewichtete Zersiedlung" (Klassifikation nach Jaeger/Schwick 2014, modifiziert}

Kartengrundlage:
ATKIS Basis-DLM (BayLDBV 2020)

(www.Idbv.bayern.de)

Beschäftigtenstatistik (LfStat 2018)

Bevölkerungsstatistik (LfStat 2018)

(www.statistik.bayern.de)

Einwohnerdaten Zensus 2011 im Gitter

(StÄdBuL 2015)

Geographisches Gitter (BKG 2018)

Bayerische Straßenbauverwaltung - BAYSIS

(www.baysis.bayern.de)

VG 250 Gebietsstand 2018 (BKG 2018)

VG 250 Gebietsstand

nach Schwarzak/Behnisch 2017)

Entwurf und Kartographie: J. C. Peters

Institut für Geographie und Geologie

JMU Würzburg

10.08.2020

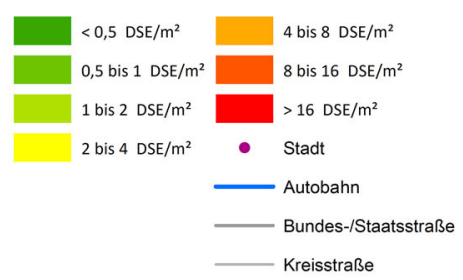

Abbildung 2 Gewichtete Zersiedelung (500-m-Raster) im Landkreis Rhön-Grabfeld

der Anordnung und Zusammensetzung dieser Landschaftselemente (Walz 2013: 12).

Zur Bestimmung der Freiraumstruktur liegt eine Vielzahl möglicher Indikatoren vor, die unterschiedliche Komponenten eines Landschaftselements beschreiben können, wie etwa Größe, Form, Lage, Umfang (Walz 2013: 16ff.). Um Lücken in den bestehenden Systemen sowie der amtlichen Flächenstatistik zu schließen, wurde der IÖR-Monitor am Leibniz-Institut für ökologische Raumentwicklung entwickelt. ${ }^{15}$ Datengrundlage des IÖR-Monitors sind topographische Geobasisdaten (ATKIS Basis-DLM), amtliche Statistikdaten und Geofachdaten (Meinel/Schumacher 2010: 186). Die Unterthemen Flächenanteile, Randdichten und Landschaftszerschneidung werden als geeignete Ansätze zum Freiraummonitoring angesehen. In vorliegender

\footnotetext{
15 Vgl. IÖR-Monitor (Stand 2019): https://www.ioer-monitor.de/ fileadmin/user_upload/monitor/pdf/Indikatoren_IOER-Monitor. pdf (09.02.2021).
} 


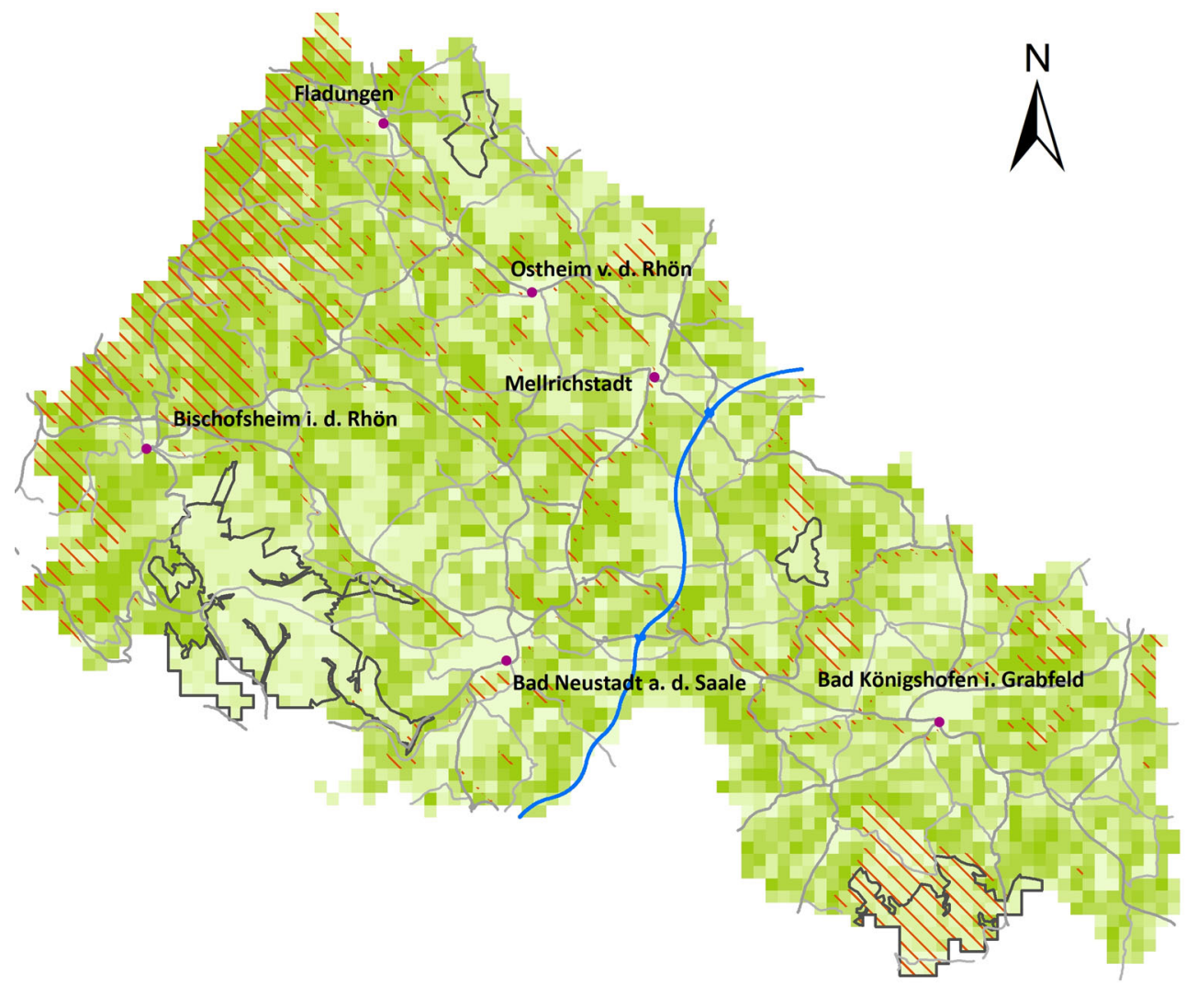

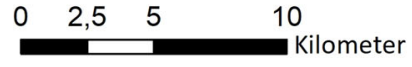

Kartengrundlage:

ATKIS Basis-DLM (BayLDBV 2020)

(www.ldbv.bayern.de)

Geographisches Gitter (BKG 2018)

Bayerische Straßenbauverwaltung - BAYSIS

(www.baysis.bayern.de)

Schutzgebiete (LfU 2019)

(www.lfu.bayern.de)

VG 250 Gebietsstand 2018 (BKG 2018)

(www.bkg.bund.de)

Entwurf und Kartographie: J. C. Peters Institut für Geographie und Geologie JMU Würzburg

10.08.2020
Klassifikation des Indikators "Freiraumstruktur"

(eigene Berechnungen)

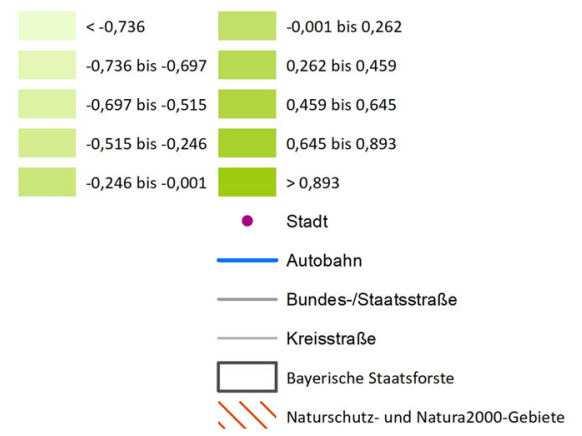

Abbildung 3 Freiraumstruktur (500-m-Raster) im Landkreis Rhön-Grabfeld

Untersuchung wurden die Flächenanteile nach IÖR-Monitor um die zusätzlichen Nutzungsarten des ATKIS BasisDLM ergänzt, sodass 15 Nutzungsarten unterschieden werden. Diese wurden anhand des Shannon-Diversity-Index und Shannon-Evenness-Index hinsichtlich ihrer Strukturvielfalt analysiert (Job/Langer/Metzler 2002: 235). Dabei untersucht der Shannon-Diversity-Index die Konfiguration der Fläche, sprich wie viele verschiedene Nutzungseinheiten sind in einer Untersuchungseinheit vorhanden, und der Shannon-Evenness-Index die Komposition, sprich wie gleichmäßig die Nutzungsklassen verteilt sind. Die Ergebnisse wurden standardisiert und zum Indikator Flächennutzungsvielfalt gemittelt. Die Randdichten (Gehölz dominiert, Gewässerrand) wurden nach IÖR-Methode berechnet und ihre Länge auf die 500-m-Rasterzellen aggregiert.

Zur Bestimmung der Landschaftszerschneidung wurde die Verkehrslinienlänge angewendet (Jaeger 2003: 11). Straßen mit einer Zerschneidungswirkung (in dieser Untersuchung Straßen >5,5 Meter) wurden als Trennelemente in der Landschaft betrachtet und, entsprechend den Randdich- 
ten, ihre Länge auf das 500-m-Raster aggregiert. Aufgrund der rasterbasierten Methodik konnte auf eine Einbeziehung der Verkehrsliniendichte verzichtet werden. Abschließend wurden die drei Indikatoren standardisiert und ein Mittelwert aus den standardisierten Variablen gebildet (Stein/ Walz 2018: 156 ff.), der je nach Höhe des Werts den Strukturreichtum beschreibt. ${ }^{16}$

In Abbildung 3 wird der Indikator „Freiraumstruktur“ in einem 500-m-Raster präsentiert. Die Ergebnisse wurden nach der ArcGIS-Klassifizierung „Quantile“ in zehn Klassen strukturiert, wobei alle Klassen die gleiche Anzahl von Merkmalen besitzen. Je höher der Wert ist, desto strukturreicher ist der Freiraum. Entsprechend sind niedrige Werte als strukturschwacher Freiraum anzusehen. Zusätzlich zur „Freiraumstruktur“ sind die Bayerischen Staatsforste in gemeindefreien Gebieten sowie Naturschutz- und Natura2000-Gebiete dargestellt. Insbesondere im Bereich der gemeindefreien Gebiete herrscht geringer Strukturreichtum. Dies ist auf die Funktion der Flächen als wirtschaftlich genutzte Forste zurückzuführen. Insbesondere der Burgwallbacher Forst im Südwesten, der Sulzfelder Forst und der Bundorfer Forst im Südosten sowie der Mellrichstadter Forst im Norden fallen hier auf. Ausschlaggebend für die Werte ist, dass lediglich eine geringe Landschaftszerschneidung als positiver Teilindikator vorliegt, demgegenüber jedoch nur geringe Randdichten und eine niedrige Flächennutzungsvielfalt als negative Teilindikatoren bestehen. ${ }^{17}$

Besonders strukturreich sind die Flächen im Westen und Nordwesten des Landkreises, die zu den Kernzonen des Biosphärenreservats Rhön gehören oder sich in unmittelbarer Umgebung befinden. Dort verfügt die Landschaft kulturgeschichtlich bedingt über einen hohen Grad an Offenheit (artenreiche Bergwiesen extensiver Bewirtschaftung). $\mathrm{Zu}$ sehen sind Maßnahmen des Biosphärenreservats, die unter anderem auf einen mosaikartigen Wechsel von strukturreichem Grünland sowie geschlossene Waldflächen und klein parzellierte Grünlandsysteme in Steillagen abzielen. ${ }^{18}$ Tendenziell werden offene Freiflächen positiver bewertet, da dort häufiger als in Waldgebieten verschiedene Landnutzungstypen auf engem Raum vorkommen. Hier sei darauf verwiesen, dass die Aussagekraft des Indikators im Hin-

\footnotetext{
16 Für eine genauere Erläuterung der Konzeption und Berechnung des Indikators „Freiraumstruktur" wird auf das Online Supplementary Material verwiesen (https://doi.org/10.14512/rur.40).

17 Dabei wird die Waldstruktur im engeren Sinne nicht tiefergehend untersucht. Denn dies ist aufgrund der geringen Differenzierung von Waldflächen im Objektartenkatalog des zugrunde liegenden ATKIS Basis-DLM kaum zufriedenstellend möglich.

18 Vgl. Verein Natur und Lebensraum Rhön 2020: https://www. biosphaerenreservat-rhoen.de/natur/landschaftsbild/ landschaftsbild-und-landnutzungswandel/ (09.02.2021).
}

blick auf den ökologischen Wert der Flächen stark auf der Landschaftsstruktur beruht. Eine aktuelle Studie verdeutlicht jedoch die Bedeutung von Komposition und Konfiguration naturnaher Flächen für den Erhalt funktionaler Biodiversität in agrarisch geprägten Landschaftsräumen (Martin/ Dainese/Clough et al. 2019: 1092).

\subsection{Fernerkundungsbasierte Analyse der Bodenversiegelung}

Eine Herausforderung in der Erfassung der tatsächlich versiegelten Fläche war für lange Zeit der Mangel an einer einheitlichen Methodik, die in der Lage ist, flächendenkende Informationen zu liefern und dabei regionalen und auf die Bevölkerungsdichte bezogenen Unterschieden gerecht zu werden. Einen frühen Versuch zur Nutzung fernerkundlich generierter Daten testeten Kreuz und Wenng (1990), indem in einem Bottom-Up-Ansatz die Versiegelungsgrade von acht Gemeindetypen anhand von hoch aufgelösten Luftbilddaten manuell ausgewertet wurden. Zur automatisierten Fortschreibung von gemeindebasierten Versiegelungsgraden eignete sich dieser Ansatz nicht. Dies wandelte sich mit der Änderung der Datenpolitik des „United States Geological Surveys" (USGS) bezüglich der Landsat-Daten im Jahr 2009, einhergehend mit der steten Verbesserung der Rechnerkapazitäten. Im Folgenden wurden verschiedene Verfahren zur Abschätzung der Bodenversiegelung vorangetrieben (Bauer/Loffelholz/Wilson 2008; Weng/Lu 2008; Esch/Himmler/Schorcht et al. 2009). Die fernerkundliche Datengrundlage verbessert sich seitdem stetig, insbesondere seit dem Start von Landsat- 8 und der Sentinel-Serie (vor allem Sentinel-2). Dennoch genügen die existierenden Verfahren noch immer nicht den Ansprüchen eines belastbaren kleinräumigen Monitorings (Meinel/Reiter 2019: 169). Die Gründe dafür sind systematisch. Fehlende Datensicherheit durch Wolkenbedeckung, räumliche Auflösungen, die keine exakte Abgrenzung einzelner Siedlungselemente erlauben, Überdeckung von versiegelter Fläche durch hohe Vegetation oder Schattenwurf sind neben methodischen Herausforderungen nur einige Faktoren, die ein kleinräumiges Monitoring erschweren.

Im Jahr 2007 veröffentlichte der Freistaat Bayern die Studie „Satellitengestützte Erfassung der Bodenversiegelung in Bayern" (LfU 2007), in der erstmals auf der Basis von Fernerkundungsdaten flächendeckend die Bodenversiegelung auf Gemeindeebene ermittelt wurde. Zur Anwendung kam ein Ansatz des maschinellen Lernens, indem Reflexionsspektren von Landsat-Daten des Jahres 2000 auf höher aufgelöste Versiegelungsinformationen trainiert und dann auf die gesamte Siedlungs- und Verkehrsfläche übertragen werden konnten (Esch/Himmler/Schorcht et al. 2009: 1682 ff.). Anschließend wurden die fernerkundlich 


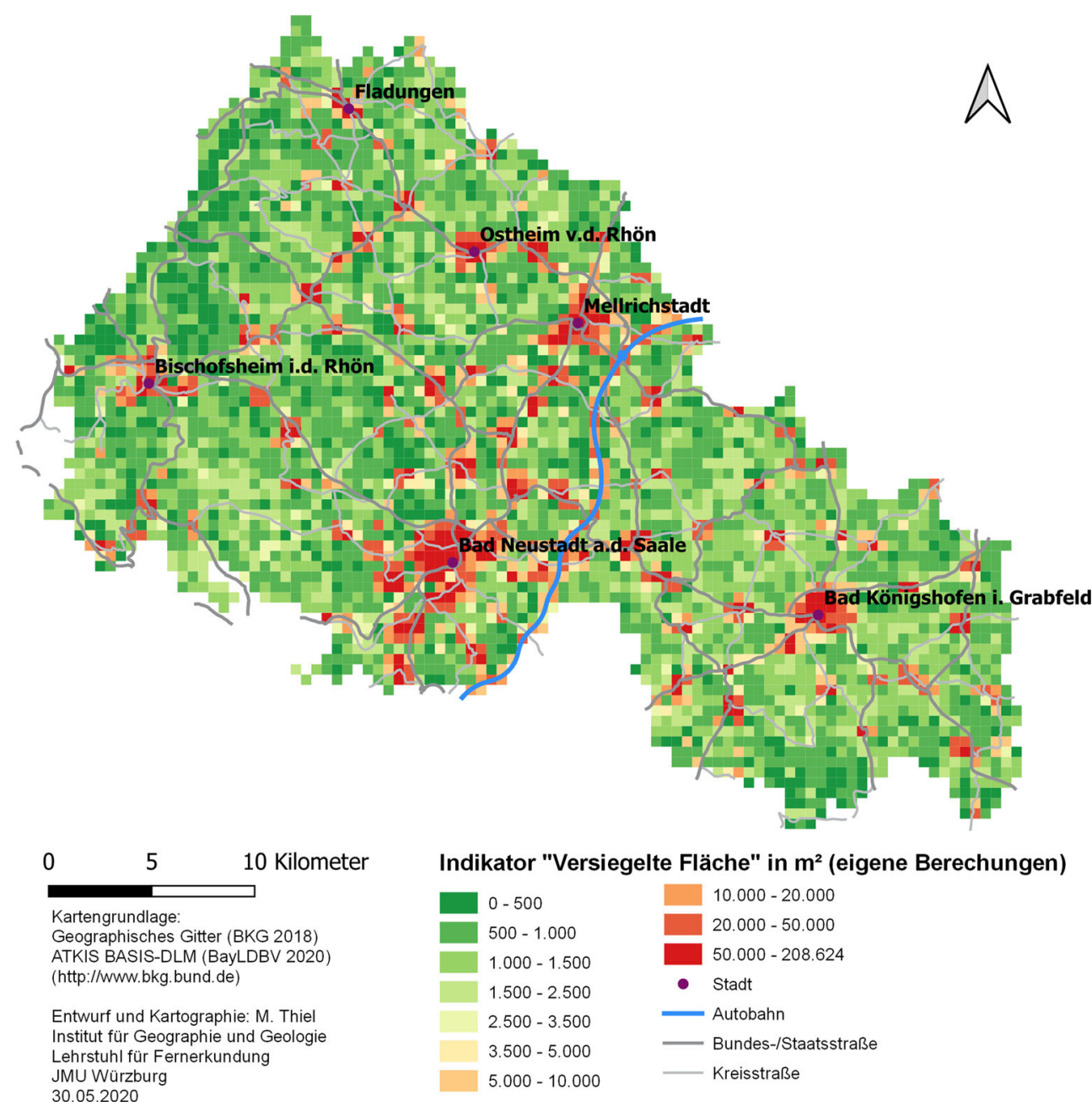

Abbildung 4 Versiegelte Fläche (500-m-Raster) im Landkreis Rhön-Grabfeld

erzielten Ergebnisse durch eine GIS-Verschneidung mit den Versiegelungsanteilen von Straßen und Bahnlinien ergänzt. Die Erfassung dieser teilweise schmalen linienhaften Elemente war mit den verwendeten 30 Meter aufgelösten Landsat-Daten nicht zufriedenstellend möglich. Dieser Ansatz wurde im Jahr 2016 mit Landsat-Daten des Jahres 2015 erneut angewendet (LfU 2017). Als Ergebnis liefern diese Studien pro Gemeinde den Versiegelungsgrad innerhalb der Siedlungs- und Verkehrsfläche sowie die versiegelte Fläche pro Einwohner (LfU 2017: 30). Für die Gemeinde Bischofsheim lässt sich beispielhaft für das Jahr 2000 (bzw. 2015) ein Versiegelungsgrad der Siedlungs- und Verkehrsfläche von 45,3\% (44,6\%) sowie eine Pro-Kopf-Versiegelung von $561 \mathrm{~m}^{2}\left(355 \mathrm{~m}^{2}\right)$ nennen. Der Landkreis Rhön-Grabfeld weist für das Jahr 2000 (2015) einen Versiegelungsgrad von 50,9\% (50,0\%) und eine Pro-Kopf-Versiegelung von $547 \mathrm{~m}^{2}\left(389 \mathrm{~m}^{2}\right)$ auf.

Einen weiteren konsistenten Datensatz zur Flächenversiegelung liefert seit 2016 der „Copernicus Land Monito- ring Service“. Dieser europäische Dienst stellt Daten für die Jahre 2006, 2009, 2012 und 2015 bereit (Langanke 2016: $12 \mathrm{ff}$.). Entgegen dem Ansatz von Esch, Himmler, Schorcht et al. (2009) werden in diesem Ansatz jedoch kleine Straßen und andere linienhafte Verkehrselemente nicht gesondert eingebunden. Somit sind die beiden Datensätze trotz ähnlicher Ausgangsdatenlage nicht miteinander vergleichbar. Für die Gemeinde Bischofsheim wird für die einzelnen Zeitschnitte eine versiegelte Fläche von 125,8 ha (2006), 125,8 ha (2009), 128,2 ha (2012) sowie 127,6 ha (2015) ermittelt. Mit einer Zunahme der Versiegelung von 1,8 ha liegt Bischofsheim über dem Median im Landkreis. $15 \mathrm{Ge}$ meinden zeigen eine höhere, 21 Gemeinden eine geringere Zunahme.

Da in der von Esch, Himmler, Schorcht et al. (2009) praktizierten Methodik die Versiegelungsinformationen lediglich auf der Basis der Gemeinden vorliegen, werden in der hier vorgeschlagenen Vorgehensweise die rasterbasierten Daten von Langanke (2016) verwendet, die zudem ak- 
tueller sind. Um die darin fehlende Berücksichtigung linienhafter Verkehrselemente außerhalb der Siedlungskörper auszugleichen, werden die frei verfügbaren Daten aus OpenStreetMap (OSM) eingebunden. Hierzu werden für jedes 500-m-Rasterelement außerhalb der Siedlungskörper die Straßen und Wege (in OSM: „ways") heruntergeladen und entsprechend ihrer Kennung (in OSM: „key“) mit einer Breite versehen. Die Breite der Straßen und Wege entspricht der vom Landesamt für Umwelt (LfU 2017: 29f.) angegebenen Breite, die wiederum auf der Grundlage der Autobahndirektion Südbayern basieren.

Im Indikator „Versiegelte Fläche“ (vgl. Abbildung 4) sind insbesondere die Siedlungsräume deutlich zu erkennen. Die am stärksten versiegelten Rasterzellen liegen in den Städten des Landkreises und erreichen zum Teil Versiegelungsanteile von $80 \%$, was über $200.000 \mathrm{~m}^{2}$ versiegelter Fläche in einer $500 \mathrm{~m}$ breiten Rasterzelle entspricht. Durch die Integration der Straßen und Wege aus OSM zeigen sich zudem Unterschiede der Flächenversiegelung in den siedlungsfernen Lagen. Die auf 20 Meter aufgelösten Rasterdaten von Langanke (2016) werden ebenfalls auf 500x500 Meter aggregiert und so mit den Informationen aus OSM verschnitten.

Neben den in der Karte eingezeichneten Bundesstraßen und Autobahnen stechen besonders die räumlichen Muster weiterer Verkehrsachsen einschließlich der dadurch verbundenen Siedlungen hervor, wie etwa die Staatsstraße 2445 zwischen Mellrichstadt und Bad Neustadt an der Saale sowie die Staatsstraße 2289 zwischen Nordheim vor der Rhön und Bischofsheim in der Rhön. Ein Vorteil der rasterbasierten Darstellung der Flächenversiegelung liegt vor allem darin, Vergleiche zu den jeweiligen Anteilen der Siedlungsund Verkehrsfläche (vgl. Abbildung 1) vornehmen zu können. Auch wenn sich die Skalen der jeweiligen Farbdarstellungen unterscheiden, zeigt sich, dass selbst kleinere ländliche Gemeinden mit geringen SuV-Anteilen zum Teil durch ein relativ hohes Maß an Flächenversiegelung geprägt sind.

\subsection{Analyse und Monetarisierungsansätze von Ökosystemleistungen}

Mit dem „Millennium Ecosystem Assessment“ (MEA 2005) setzt eine intensive Auseinandersetzung und zunehmende Erweiterung des Konzepts der Ökosystemleistungen ein. Das vorrangige Ziel dieses Konzeptes ist es, Ökosysteme als grundlegenden Wohlfahrtsfaktor, als Quelle für menschliches Wohlbefinden zu identifizieren, zu monetarisieren und somit besser zu schützen. Zahlreiche Ökosystemleistungen sind grundsätzlich als öffentliche Güter anzusehen, für die keine direkten Marktmechanismen greifen. Soll eine Monetarisierung von Ökosystemleistungen betrieben werden, kann diese allenfalls indirekt über hypothetische
Märkte geschehen. Diese monetäre Bewertung dient dann als Basis für öffentliche Entscheidungen und individuelle Verhaltensweisen (Goulder/Kennedy 2011: 15; TEEB 2012: 21; van Beukering/Brouwer/Koetse 2015: 90). Die Klassifikation des „Millennium Ecosystem Assessment“ unterteilt Ökosystemleistungen in Versorgungsleistungen, Regulierungsleistungen, kulturelle Leistungen und unterstïtzende Leistungen (MEA 2005: 40).

Die Schädigung regionaler und globaler Ökosysteme impliziert im Konzept der Ökosystemleistungen entsprechende gesellschaftliche Kosten. Bezüglich der Freiflächeninanspruchnahme ist daher primär ein sparsamer Umgang mit unbebauten Flächen anzustreben, denn eine spätere Flächenentsiegelung kann die Ökosystemleistungen nur sehr langfristig und unzureichend wieder herstellen. Die Flächeninanspruchnahme durch Versiegelung kann je nach Größe und Lage der Fläche ganz unterschiedliche Ökosystemleistungen beeinträchtigen. Eine Flächenversiegelung hat nicht nur Auswirkungen auf die Fläche selbst, sondern auch auf weiter entfernte Flächen. Dies wird bei der Regulierung des Wasserhaushaltes besonders deutlich, wo versiegelte Flächen zu verstärkten Hochwasserspitzen und entsprechenden Schäden in entfernteren Gebieten führen können (Graves/Morris/Deeks et al. 2015: 409). Der weitere Verlust von Ökosystemleistungen durch Flächenversiegelung umfasst Versorgungsleistungen (z. B. agrarische Produktion), Regulierungsleistungen (z. B. Hochwasserschutz), kulturelle Leistungen (z. B. Erholung) und unterstützende Leistungen (z. B. Bodenbildung). In einem konzeptionellen Rahmen werden nachfolgend unterschiedliche Kriterien zur Bewertung der Ökosystemleistungen von Flächen präsentiert, die sodann beispielhaft monetarisiert werden.

Basierend auf Flächen des Bayerischen Vertragsnaturschutzprogrammes (VNP) werden Ökosystemleistungen von Grün- und Ackerland im Landkreis Rhön-Grabfeld gegenübergestellt (vgl. Rathmann/Dubrow/Eberhardt 2019). Am Beispiel des Grünlandumbruchs kann eine Monetarisierung von VNP-Flächen gut illustriert werden. Die Klimaschutzfunktion lässt sich dabei über die Treibhausgasspeicherung pro Jahr operationalisieren. In der landwirtschaftlichen Nutzung werden insbesondere durch Grünlandumbruch, Pflanzenbau, Düngung und Tierhaltung Treibhausgase freigesetzt (Götzl/Schwaiger/Sonderegger et al. 2011: 31; Albert/Burkhard/Daube et al. 2015: 32). Die Übergangszeit bei Landnutzungsänderungen, bis sich ein neuer Systemzustand einstellt, liegt bei etwa 20 Jahren (Gensior/Dunger/Stürmer et al. 2018: 528). Daher wird die Gesamtänderung durch 20 dividiert, um eine jährliche Veränderung $\mathrm{zu}$ erhalten, die einen Vergleich mit anderen Ökosystemleistungen erst ermöglicht. Für den Untersuchungsraum ergeben sich dann folgende Werte: Unter einem Hektar Grünland lassen sich durchschnittlich etwa 
393 t CO$_{2}$ sequestrieren, der vergleichbare Wert für Ackerland liegt nur bei $263 \mathrm{t} \mathrm{CO}_{2}$; das entspricht einem Anteil von $67 \%$. Der Verlust an der $\mathrm{CO}_{2}$-Speicherkapazität, der demnach durch Umwandlung generiert wird, beträgt $130 \mathrm{t}$ $\mathrm{CO}_{2} /$ ha.

Die Monetarisierung der Ökosystemleistung Klimaschutz wird in Werten für vermiedene Kohlenstoffemissionen dargestellt (Remme/Edens/Schröter et al. 2015: 120). Dies basiert auf den Schadenskosten der Methodenkonvention 3.0 des Umweltbundesamtes, wobei ein Wert von 180 Euro/t $\mathrm{CO}_{2}$ äquivalent für das Jahr 2016 bei einer Zeitpräferenzrate von einem Prozent empfohlen wird (Matthey/ Bünger 2018: 9). Dabei werden die Klimakostensätze für die Jahre 2030 und 2050 angegeben. Um die Schadenskosten für das Jahr 2018 zu ermitteln, wird zwischen den Werten für 2016 und 2030 linear interpoliert. Der Schadenskostensatz für 2018 beträgt etwa 185 Euro. Zur Preisbereinigung des Kostensatzes wird der Verbraucherpreisindex 2018 des Statistischen Bundesamts verwendet. ${ }^{19}$ Die Klimaregulierungsleistung in Euro/ha/Jahr wird dadurch berechnet, dass die jährliche Änderung des Kohlenstoffs im Boden (infolge der Umwandlung von Grün- in Ackerland) mit dem erwähnten Kostensatz multipliziert wird. Grünland stellt demnach eine Klimaschutzleistung mit einem Wert von durchschnittlich 72.765 Euro/ha/Jahr bereit, Ackerland hingegen lediglich 48.641 Euro/ha/Jahr. ${ }^{20}$ Dieser Ansatz zeigt beispielhaft die Höhe einer Ökosystemleistung, die sich bei einer Nutzungsänderung einer Fläche ergibt, wobei eine Flächenversiegelung die Klimaregulierungsleistung weitgehend ausschließt.

Noch deutlicher werden die Änderungen der Ökosystemleistungen am Beispiel der Umweltleistung „Erhalt der Biodiversität", welche immer in der relativen Standortlage bewertet werden muss. Eine Versiegelungsmaßnahme führt zu drastischen Änderungen der Biodiversität. Nicht nur am Standort selbst, sondern durch eine Habitatzerschneidung sind auch negative Auswirkungen auf die Umgebung gegeben. Die Ökosystemleistungen einer Fläche lassen sich dabei nicht nur absolut angeben, da diese immer relational von der Lage und Größe der jeweiligen Fläche abhängen. Ökosystemleistungen bezüglich der absoluten Lage einer Fläche lassen sich beispielsweise über das Naturraumpotenzial spezifizieren. Die relative Lage einer Fläche bezieht

\footnotetext{
19 https://www.destatis.de/DE/ZahlenFakten/

GesamtwirtschaftUmwelt/Preise/Verbraucherpreisindizes/

Tabellen_/VerbraucherpreiseKategorien.html?cms_gtp=

145114_list\%253D2\%2526145112_list\%253D2\%2526145110

_slot\%253D2 (09.02.2021).

20 Für eine detailliertere ÖSL-Bewertung aus regionaler Perspektive sei auf die Machbarkeitsstudie des Projekts „Naturkapital Bayern“ (Rathmann/Dubrow/Eberhardt 2019) verwiesen.
}

sich beispielsweise auf die Nähe von Siedlungen. Die Habitatfunktion einer Fläche ist hingegen für viele Arten in siedlungsfernen Lagen besser - dies gilt insbesondere für hoch spezialisierte Arten, beispielsweise Schmetterlinge. Einen Einfluss hat auch die Siedlungsstruktur. Eine stark gestreute Siedlung erleichtert die Ausbreitung von Generalisten oder Neophyten, spezialisierte Arten profitieren hingegen von kompakteren Siedlungen (Tobias/Ströbele/Nobis et al. 2016: 6).

Bei der Monetarisierung von durch Entsiegelungsmaßnahmen gewonnenen Ökosystemleistungen ist der zeitliche Bezugsrahmen entscheidend für die Höhe der Ökosystemleistungen, wie am Beispiel der „Klimakosten“ schon gezeigt wurde. Schließlich benötigt eine entsiegelte Fläche eine gewisse Zeit, um die Ökosystemleistungen voll zur Entfaltung zu bringen. Nach 15 Jahren lassen sich die bodenphysikalischen Eigenschaften für eine agrarische Nutzung wiederherstellen, die ursprünglichen natürlichen Eigenschaften sind damit aber noch nicht erreicht (Tobias/Conen/Duss et al. 2018: 2015). Die Übergangszeit beim Grünlandumbruch bezüglich des geänderten Kohlenstoffhaushaltes wird mit 20 Jahren veranschlagt (Gensior/Dunger/Stürmer et al. 2018: 582). Gleichzeitig ist zu berücksichtigen, dass die Ökosystemleistungen über die eigentliche Fläche hinausreichen können. Auf der Mikroebene wirkt eine Entsiegelung unmittelbar beispielsweise als Habitatgewinn, auf der Mesoebene kann sich eine verbesserte klimaregulierende Leistung auswirken und auf der Makroebene sind Einflüsse auf den Wasserhaushalt durch eine wiedergewonnene Versickerung erkennbar. Weiter ist ein Skaleneffekt zu beachten: Bestimmte Ökosystemleistungen sind unmittelbar an die Größe der Fläche gebunden, was sich für artspezifische Habitate deutlich belegen lässt. Regulierende Ökosystemleistungen wirken aber auf jeder Flächengröße.

\section{Diskussion}

Die Akademie für Raumentwicklung in der Leibniz-Gemeinschaft (ARL) beschäftigt sich wegen der Brisanz der Freiflächeninanspruchnahme schon seit Mitte der 1980erJahre mit diesem Thema (vgl. ARL 1987; ARL 1999). Dabei wurde immer wieder die Bedeutung einer wirksamen Steuerung betont: „Die Flächenhaushaltspolitik (FHP) ist ein unumgänglicher Weg zu haushälterischem Umgang mit der Ressource Fläche. Es geht ihr einerseits um eine drastische Begrenzung der Flächeninanspruchnahme (Mengenziel), andererseits um den Ausgleich und die Wiederherstellung ökologischer Funktionen (Qualitätsziel)“ (ARL 2004: 1). Der in diesem Beitrag vorgestellte Ansatz zur differenzierten Bewertung von Flächeninanspruchnahme und 
-versiegelung erfüllt diesen von der ARL formulierten Anspruch. Mithilfe der am Beispiel des Kreises Rhön-Grabfeld aufgezeigten Methoden wird eine Orientierung für die notwendige Weiterentwicklung des staatlichen Flächenmonitorings in Ergänzung zu den bestehenden Indikatoren der Flächenstatistik dargestellt. Es gilt hierbei insbesondere bei der Einbeziehung des Ökosystemleistungsansatzes, von der exemplarischen Anwendung hin zu einem für das kontinuierliche Flächenmonitoring geeigneten, realistischen Analyserahmen zu kommen.

Die Weiterentwicklung dieses Flächenmonitorings ist nicht nur als Beurteilungsgrundlage für politisch-gesellschaftliche Aushandlungsprozesse von Bedeutung. Vielmehr ist es erstrebenswert, die in Rechtsgrundlagen und Raumordnungsplänen verwendeten Begrifflichkeiten wie „Bodenversiegelung“ (vgl. § 1a Abs. 2 S. 1 BauGB, § 5 BBodSchG $^{21}$ ), „Zersiedelung“ (vgl. LEP Bayern ${ }^{22}$, Kapitel 3.3) oder auch „Freiraumverbund“ und „Landschaftszerschneidung" (vgl. Art. 6 Abs. 2 Nr. 3 BayLplG; § 2 Abs. 2 Nr. 2 ROG) zukünftig besser zu operationalisieren und somit erst messbar zu machen. Dass diese bisher noch nicht ausreichend einbezogen werden, zeigt beispielsweise die fehlende Berücksichtigung im 18. Bayerischen Raumordnungsbericht (StMWi 2019).

Mit den aktuellen politischen Debatten zum Flächensparen in Bayern gewinnt auch die Thematik der Flächenentsiegelung an politischer Bedeutung. Dies sollte als Anlass gesehen werden, die Wirkung potenzieller Entsiegelungsmaßnahmen genauer zu untersuchen. Neben den durch Umbaubzw. Entsiegelungsmaßnahmen direkt entstehenden Kosten, die etwa durch die bestehende Förderinitiative der Städtebauförderung adressiert werden, könnte auch der potenzielle Nutzen durch die (Wieder-)Herstellung bestimmter flächenbezogener Ökosystemleistungen künftig in die Betrachtung mit einbezogen werden.

Insgesamt sind Strategien zur dauerhaften Begrenzung der Flächeninanspruchnahme und zur konsequenten Sicherung bedeutsamer Freiräume nötig, die langfristig in einer „Flächenkreislaufwirtschaft“ münden, was auch durch den Klimaschutzplan 2050 der Bundesregierung (BMU 2019: $67 \mathrm{f}$.) sowie das „Netto-Null“-Flächenziel der Europäischen Kommission (2011: 18) wiedergegeben wird. Wesentliche Bausteine umfassen neben der Weiterentwicklung des Flächenmonitorings eine Verbesserung der Instrumente der Innenentwicklung, neue Sicherungsinstrumente für landschaftliche und landwirtschaftliche Freiflächen sowie

21 Bundes-Bodenschutzgesetz vom 17. März 1998, das zuletzt durch Artikel 3 Absatz 3 der Verordnung vom 27. September 2017 geändert worden ist.

22 Landesentwicklungsprogramm Bayern, Stand 1. Januar 2020. die Entwicklung und Zuweisung von Flächenkontingenten (Adrian/Bock/Bunzel et al. 2018: $44 \mathrm{ff}$;; ARL 2018: 6: ff.). Der vorliegende Beitrag nennt methodische Erweiterungsansätze, die politische und planerische Strategien als Beitrag für eine nachhaltige Raumentwicklung unterstützen können.

Danksagung Wir danken für die Förderung im Rahmen des Forschungsprojekts „Naturkapital Bayern“ (2019) des Bayerischen Landesamts für Umwelt im Auftrag des Bayerischen Staatsministeriums für Umwelt und Verbraucherschutz.

\section{Literatur}

Adrian, L.; Bock, S.; Bunzel, A.; Preuß, T.; Rakel, M. (2018): Instrumente zur Reduzierung der Flächeninanspruchnahme. Aktionsplan Flächensparen. Dessau-Roßlau. = UBA-Texte 38/2018.

Albert, C.; Burkhard, B.; Daube, S.; Dietrich, K.; Engels, B.; Frommer, J.; Götzl, M.; Gret-Regamey, A.; Job-Hoben, B.; Keller, R.; Marzelli, S.; Moning, C.; Müller, F.; Rabe, S.-E.; Ring, I.; Schwaiger, E.; Schweppe-Kraft, B.; Wüstemann, H. (2015): Empfehlungen zur Entwicklung bundesweiter Indikatoren zur Erfassung von Ökosystemleistungen. Bonn. = BFN-Skripten 410.

ARL - Akademie für Raumforschung und Landesplanung (Hrsg.) (1987): Flächenhaushaltspolitik. Ein Beitrag zum Bodenschutz. Hannover. = Forschungs- und Sitzungsberichte der ARL 173.

ARL - Akademie für Raumforschung und Landesplanung (Hrsg.) (1999): Flächenhaushaltspolitik. Feststellungen und Empfehlungen für eine zukunftsfähige Raum- und Siedlungsentwicklung. Hannover. $=$ Forschungs- und Sitzungsberichte der ARL 208.

ARL - Akademie für Raumforschung und Landesplanung (Hrsg.) (2004): Flächenhaushaltspolitik. Ein Beitrag zur nachhaltigen Raumentwicklung. Hannover. $=$ Positionspapier aus der ARL 58.

ARL - Akademie für Raumforschung und Landesplanung (Hrsg.) (2018): Begrenzung der Flächenneuinanspruchnahme in Bayern. Hannover. $=$ Positionspapier aus der ARL 111.

Baldenius, T.; Kohl, S.; Schularick, M. (2019): Die neue Wohnungsfrage. Gewinner und Verlierer des deutschen Immobilienbooms. Bonn. http://www.macrohistory.net/ wp-content/uploads/2019/06/Die-neue-Wohnungsfrage- . pdf (08.02.2021).

Bauer, M. E.; Loffelholz, B. C.; Wilson, B. (2008): Estimating and mapping impervious surface area by regression analysis of landsat imagery. In: Weng, Q. (Hrsg.): Remote Sensing of Impervious Surfaces. Boca Raton, 3-19. https://doi.org/10.1201/9781420043754 
Beckmann, G.; Dosch, F. (2018a): Monitoring der Siedlungsflächenentwicklung. In: Behnisch, M.; Kretschmer, O.; Meinel, G. (Hrsg.): Flächeninanspruchnahme in Deutschland. Auf dem Wege zu einem besseren Verständnis der Siedlungs- und Verkehrsflächenentwicklung. Berlin, 3-24. https://doi.org/10.1007/978-3-66250305-8_1

Beckmann, G.; Dosch, F. (2018b): Das Siedlungsflächenmonitoring des Bundes zwischen Anspruch und Praxis. In: Stadtforschung und Statistik 31, 2, 13-22.

Behnisch, M.; Kretschmer, O.; Meinel, G. (Hrsg.) (2018): Flächeninanspruchnahme in Deutschland. Auf dem Wege zu einem besseren Verständnis der Siedlungs- und Verkehrsflächenentwicklung. Berlin. https://doi.org/10. 1007/978-3-662-50305-8

BMU - Bundesministerium für Umwelt, Naturschutz und nukleare Sicherheit (2019): Klimaschutzplan 2050. Klimaschutzpolitische Grundsätze und Ziele der Bundesregierung. Berlin.

Bräuninger, M.; Schnaars, P. (2017): Bautätigkeit in den Bundesländern bis 2017. In: Wirtschaftsdienst 97, 3, 228-230. https://doi.org/10.1007/s10273-017-2112-7

Bundesregierung (2002): Perspektiven für Deutschland. Unsere Strategie für eine nachhaltige Entwicklung. Berlin.

Bundesregierung (2016): Deutsche Nachhaltigkeitsstrategie. Neuauflage 2016. Berlin.

Esch, T.; Himmler, V.; Schorcht, G.; Thiel, M.; Conrad, C.; Wehrmann, T.; Bachofer, F.; Schmidt, M.; Dech, S. (2009): Large-area assessment of impervious surface based on integrated analysis of single-date Landsat-7 images and geospatial vector data. In: Remote Sensing of Environment 113, 8, 1678-1690. https://doi.org/10.1016/ j.rse.2009.03.012

Europäische Kommission (2011): Mitteilung der Kommission an das Europäische Parlament, den Rat, den Europäischen Wirtschafts- und Sozialausschuss und den Ausschuss der Regionen. Fahrplan für ein ressourcenschonendes Europa. KOM(2011) 571 endgültig. Brüssel.

Fina, S. (2018): Ist die Konfiguration und Komposition der Flächennutzung messbar? In: Behnisch, M.; Kretschmer, O.; Meinel, G. (Hrsg.): Flächeninanspruchnahme in Deutschland. Auf dem Wege zu einem besseren Verständnis der Siedlungs- und Verkehrsflächenentwicklung. Berlin, 111-130. https://doi.org/10.1007/978-3662-50305-8_7

Gensior, A.; Dunger, K.; Stümer, W.; Döring, U. (2018): Übersicht (CRF Sektor 4). In: Umweltbundesamt (Hrsg.): Berichterstattung unter der Klimarahmenkonvention der Vereinten Nationen und dem Kyoto-Protokoll 2018. Nationaler Inventarbericht zum Deutschen Treibhausgasinventar 1990-2016. Dessau-Roßlau, 522-563. = Climate Change 12/2018.
Goetzke, R. (2018): Indikatoren zur Bewertung einer nachhaltigen und klimawandelgerechten Siedlungsentwicklung. In: Behnisch, M.; Kretschmer, O.; Meinel, G. (Hrsg.): Flächeninanspruchnahme in Deutschland. Auf dem Wege zu einem besseren Verständnis der Siedlungs- und Verkehrsflächenentwicklung. Berlin, 131154. https://doi.org/10.1007/978-3-662-50305-8_8

Götzl, M.; Schwaiger, E.; Sonderegger, G.; Süßenbacher, E. (2011): Ökosystemleistungen und Landwirtschaft. Erstellung eines Inventars für Österreich. Wien.

Goulder, L. H.; Kennedy, D. (2011): Interpreting and estimating the value of ecosystem services. In: Kareiva, P.; Tallis, H.; Ricketts, T. H.; Daily, G. C.; Polasky, S. (Hrsg.): Natural Capital: Theory and Practice of Mapping Ecosystem Services. Oxford, 15-33. https://doi.org/ 10.1093/acprof:oso/9780199588992.001.0001

Graves, A. R.; Morris, J.; Deeks, L. K.; Rickson, R. J.; Kibblewhite, M. G.; Harris, J. A.; Farewell, T. S.; Truckle, I. (2015): The total costs of soil degradation in England and Wales. In: Ecological Economics 119, 399-413. https:// doi.org/10.1016/j.ecolecon.2015.07.026

Henger, R.; Voigtländer, M. (2019): Ist der Wohnungsbau auf dem richtigen Weg? Aktuelle Ergebnisse des IWWohnungsbedarfsmodells. Köln. = IW-Report 28/2019.

Jacoby, C. (2009): Monitoring und Evaluation von Stadt und Regionalentwicklung. Einführung in Begriffswelt, rechtliche Anforderungen, fachliche Herausforderungen und ausgewählte Ansätze. In: Jacoby, C. (Hrsg.): Monitoring und Evaluation von Stadt und Regionalentwicklung. Hannover, 1-24. = Arbeitsmaterial der ARL 350.

Jaeger, J. (2003): Landschaftszerschneidung. In: Konold, W.; Böcker, R.; Hampicke, U. (Hrsg.): Handbuch Naturschutz und Landschaftspflege. Landsberg, 1-30.

Jaeger, J.; Schwick, C. (2014): Improving the measurement of urban sprawl. Weighted Urban Proliferation (WUP) and its application to Switzerland. In: Ecological Indicators 38, 294-308. https://doi.org/10.1016/j.ecolind.2013. 11.022

Jaeger, J.; Schwick, C.; Hennig E. I.; Orlitova, E.; Soukup, T.; Nazarnia, N.; Kienast, F. (2015): Zersiedelung aus landschaftsökologischer, sozialer und siedlungstechnischer Sicht. In: Eidgenössische Forschungsanstalt für Wald, Schnee und Landschaft (Hrsg.): Von der Siedlungsentwicklung zur Landschaftsgestaltung. Birmensdorf, 15-25. = WSL Berichte 33.

Job, H.; Engelbauer, M.; Engels, B. (2019): Das Portfolio deutscher Biosphärenreservate im Lichte der Sustainable Development Goals. In: Raumforschung und Raumordnung | Spatial Research and Planning 77, 1, 57-79. https://doi.org/10.2478/rara-2019-0005 
Job, H.; Langer, T.; Metzler, D. (2002): Operationalisierung europäischer Kulturlandschaften. In: Informationen zur Raumentwicklung 4/5, 231-240.

Job, H.; Mayer, M.; Kraus, F. (2014): Die beste Idee, die Bayern je hatte: der Alpenplan. Raumplanung mit Weitblick. In: GAIA - Ecological Perspectives on Science and Society 23, 4, 335-345. https://doi.org/10.14512/ gaia.23.4.9

Job, H.; Weidlich, O.; Meyer, C. (2019): Erfahrungen zum Flächensparen aus Österreich und der Schweiz sowie Schlussfolgerungen für Bayern. In: Bayerische Akademie Ländlicher Raum (Hrsg.): Flächenverbrauch wirksam begrenzen. Wie lässt sich der Richtwert 5ha/Tag in Bayern umsetzen? München, 40-44. = Dokumentation Heft 56.

Klein, R.; Wettemann, J.; Esch, T.; Himmler, V.; Schmidt, M. (2010): Das Indikatorenset „Flächenbarometer“. Neue Methoden zur Beobachtung der Flächeninanspruchnahme in Deutschland. In: Frerichs, S.; Lieber, M.; Preuß, T. (Hrsg.): Flächen- und Standortbewertung für ein nachhaltiges Flächenmanagement. Methoden und Konzepte. Berlin, 29-43. = Beiträge aus der REFINAForschung V.

Kreuz, D.; Wenng, S. (1990): Flächennutzung, Flächennutzungswandel und Flächenversiegelung in Bayern. Studie im Auftrag des Bayerischen Staatsministeriums für Landesentwicklung und Umweltfragen. Ottobrunn.

Langanke, T. (2016): Copernicus land monitoring service - High Resolution Layer Imperviousness: Product Specifications Document. European Environmental Agency. https://land.copernicus.eu/user-corner/technical-library/ hrl-imperviousness-technical-document-prod-2015 (08.02.2021).

LfStat - Bayerisches Landesamt für Statistik (2020a): Flächenerhebung nach Art der tatsächlichen Nutzung (nach ALKIS Nutzungsarten) 2014-2018. https://www. statistikdaten.bayern.de/genesis/online?language $=$ de $\&$ sequenz $=$ tabellen\&selectionname $=33111 *(08.02 .2021)$.

LfStat - Bayerisches Landesamt für Statistik (2020b): Statistik kommunal 2019. Landkreis Rhön-Grabfeld 09673. Eine Auswahl wichtiger statistischer Daten. Fürth.

LfU - Bayerisches Landesamt für Umwelt (2007): Satellitengestützte Erfassung der Bodenversiegelung in Bayern. Augsburg.

LfU - Bayerisches Landesamt für Umwelt (2017): Satellitengestützte Erfassung der Bodenversiegelung in Bayern 2015. Augsburg.

LUBW - Landesanstalt für Umwelt, Messungen und Naturschutz Baden-Württemberg (Hrsg.) (2007): Indikatoren zur Flächeninanspruchnahme und flächensparenden Siedlungsentwicklung in Baden-Württemberg. Entwicklerversion zu den Indikatoren zur Siedlungsentwicklung. Karlsruhe.
Martin, E. A.; Dainese, M.; Clough, Y.; Báldi, A.; Bommarco, R.; Gagic, V.; Garratt, M.; Holzschuh, A.; Kleijn, D.; Kovács-Hostyánszki, A.; Marini, L.; Potts, S. G.; Smith, H.; Al Hassan, D.; Albrecht, M.; Andersson, G.; Asís, J. D.; Aviron, S.; Balzan, M. V.; Baños-Picón, L.; Bartomeus, I.; Batáry, P.; Burel, F.; Caballero-López, B.; Concepción, E.D.; Coudrain, V.; Dänhardt, J.; Diaz, M.; Diekötter, T.; Dormann, C. F.; Duflot, R.; Entling, M. H.; Farwig, N.; Fischer, C.; Frank, T.; Garibaldi, L.A.; Hermann, J.; Herzog, F.; Inclán, D.; Jacot, K.; Jauker, F.; Jeanneret, P.; Kaiser, M.; Kraus, J.; Le Féon, V.; Marshall, J.; Moonen, A.-C.; Moreno, G.; Riedinger, V.; Rundlöf, M.; Rusch, A.; Scheper, J.; Schneider, G.; Schuepp, C.; Stutz, S.; Sutter, L.; Tamburini, G.; Thies, C.; Tormos, J.; Tscharntke, T.; Tschumi, M.; Uzman, D.; Wagner, C.; Zubair-Anjum, M.; Steffan-Dewenter, I. (2019): The interplay of landscape composition and configuration: new pathways to manage functional biodiversity and agroecosystem services across Europe. In: Ecology Letters 22, 7, 1083-1094. https://doi.org/10. 1111/ele. 13265

Matthey, A.; Bünger, B. (2018): Methodenkonvention 3.0 zur Ermittlung von Umweltkosten. Kostensätze. DessauRoßlau.

MEA - Millennium Ecosystem Assessment (2005): Ecosystems and human well-being. Synthesis. Washington, D.C.

Meinel, G. (2020): Herausforderung Flächenmonitoring: Datenquellen für ein Flächeninformationssystem und was sie leisten können. In: Stadtforschung und Statistik 33, 1, 107-114.

Meinel, G.; Henger, R.; Krüger, T.; Schmidt, T.; Schorcht, M. (2020): Wer treibt die Flächeninanspruchnahme? Ein Planvergleich und deren Flächenwirkung. In: Raumforschung und Raumordnung | Spatial Research and Planning 78, 3, 233-248. https://doi.org/10.2478/rara-20200003

Meinel, G.; Reiter, D. (2019): Nutzung des Landbedeckungsmodells LBM-DE für das Flächenmonitoring Bewertung und Ergebnisse. In: Meinel, G.; Schumacher, U.; Behnisch, M.; Krüger, T. (Hrsg.): Flächennutzungsmonitoring XI. Flächenmanagement - Bodenversiegelung - Stadtgrün. Berlin, 169-179. = IÖR-Schriften 77.

Meinel, G.; Schumacher, U. (2010): Konzept, Funktionalität und erste exemplarische Ergebnisse des Monitors der Siedlungs- und Freiraumentwicklung (IÖR-Monitor). In: Meinel, G.; Schumacher, U. (Hrsg.): Flächennutzungsmonitoring II. Konzepte - Indikatoren - Statistik. Berlin, 183-200. = IÖR-Schriften 52.

Miosga, M. (2019): Richtwerte als ein Baustein einer umfassenden Strategie zur Reduzierung des Flächenverbrauchs 
und zur Stärkung der Innenentwicklung. In: Bayerische Akademie Ländlicher Raum (Hrsg.): Flächenverbrauch wirksam begrenzen. Wie lässt sich der Richtwert 5ha/Tag in Bayern umsetzen? München, 7-15. = Dokumentation Heft 56.

Penn-Bressel, G. (2009): Umweltindikatoren: Die Flächeninanspruchnahme für Siedlungen und Verkehr sowie weitere relevante Indikatoren zum Zustand von Flächen und Böden. In: Meinel, G.; Schumacher, U. (Hrsg.): Flächennutzungsmonitoring. Konzepte - Indikatoren - Statistik. Aachen, 71-104.

Peters, J. C. (2020): Integration der Landschaftsstruktur in Messung von Zersiedelung. Unveröffentlichte Masterarbeit am Institut für Geographie und Geologie der JuliusMaximilians-Universität Würzburg.

Poeplau, C.; Don, A.; Vesterdal, L.; Leifeld, J.; van Wesemael, B.; Schumacher, J.; Gensior, A. (2011): Temporal dynamics of soil organic carbon after land-use change in the temperate zone - carbon response functions as a model approach. In: Global Change Biology 17, 7, 24152427. https://doi.org/10.1111/j.1365-2486.2011.02408.x

Rathmann, J.; Dubrow, C.; Eberhardt, A. (2019): Naturkapital Bayern: Machbarkeitsstudie zur ökonomischen Bewertung von Naturkapital und Ökosystemleistungen in Bayern - Ein landnutzungs-basierter Bewertungsansatz am Beispiel des bayerischen Vertragsnaturschutzprogramms. Schlussbericht. Würzburg.

Remme, R. P.; Edens, B.; Schröter, M.; Hein, L. (2015): Monetary accounting of ecosystem services. A test case for Limburg province, the Netherlands. In: Ecological Economics 112, 116-128. https://doi.org/10.1016/j. ecolecon.2015.02.015

Schiller, G.; Siedentop, S. (2005): Infrastrukturfolgekosten der Siedlungsentwicklung unter Schrumpfungsbedingungen. In: disP - The Planning Review 41, 160, 8393. https://doi.org/10.1080/02513625.2005.10556910

Schwarzak, M.; Behnisch, M. (2017): Zersiedelung in Deutschland messen und beschreiben. Anwendung der Schweizer Methode der gewichteten Zersiedelung. In: Wende, W.; Walz, U. (Hrsg.): Die räumliche Wirkung der Landschaftsplanung. Evaluation, Indikatoren und Trends. Wiesbaden, 77-96. https://doi.org/10.1007/9783-658-13556-0_6

Schwick, C.; Jaeger, J. (2010): Zersiedelung und ihre Ausprägungen in der Schweiz aus raumplanerischer Sicht. Quantitative Analyse 1935-2002. Bern.

Schwick, C.; Jaeger, J.; Kienast, F. (2011): Zersiedelung messen und vermeiden. Birmensdorf. = Merkblatt für die Praxis 47.

Siedentop, S.; Heiland, S.; Lehmann, I.; Hernig, A.; Schauerte-Lüke, N. (2007): Nachhaltigkeitsbarometer Fläche. Regionale Schlüsselindikatoren nachhaltiger Flächennut- zung für die Fortschrittsberichte der nationalen Nachhaltigkeitsstrategie - Flächenziele. Bonn. = BMVBS/BBR Forschungen 130.

Siedentop, S.; Junesch, R.; Straßer, M.; Zakrzewski, P.; Samaniego, L.; Weinert, J. (2009): Einflussfaktoren der Neuinanspruchnahme von Flächen. Bonn. = BMVBS/BBSR Forschungen 139.

Statistisches Bundesamt (2020): Anstieg der Siedlungsund Verkehrsfläche in ha/Tag. Wiesbaden. https:// www.destatis.de/DE/Themen/Branchen-Unternehmen/ Landwirtschaft-Forstwirtschaft-Fischerei/

Flaechennutzung/Publikationen/Downloads-

Flaechennutzung/anstieg-suv.html (22.01.2021).

Staub, C.; Ott, W.; Heusi, F.; Klinger, G.; Jenny, A.; Häcki, M.; Hauser, A. (2011): Indikatoren für Ökosystemleistungen: Systematik, Methodik und Umsetzungsempfehlungen für eine wohlfahrtsbezogene Umweltberichterstattung. Bern. = Umwelt-Wissen 1102.

Stein, C.; Walz, U. (2018): Indikator für ein Monitoring der landschaftlichen Attraktivität Deutschlands. In: Behnisch, M.; Kretschmer, O.; Meinel, G. (Hrsg.): Flächeninanspruchnahme in Deutschland. Auf dem Wege zu einem besseren Verständnis der Siedlungs- und Verkehrsflächenentwicklung. Berlin, 155-169. https://doi.org/10. 1007/978-3-662-50305-8_9

StMB - Bayerisches Staatsministerium für Wohnen, Bau und Verkehr (2018): So werden in Bayern Flächen geschont. Eine Richtschnur für den Wohnungs- und Städtebau. München.

StMWi - Bayerisches Staatsministerium für Wirtschaft, Landesentwicklung und Energie (Hrsg.) (2019): 18. Raumordnungsbericht. Bayern 2013-2017. München.

TEEB - The Economics of Ecosystems and Biodiversity (2012): The economics of ecosystems and biodiversity. Ecological and economic foundations. Abingdon.

Tobias, S.; Conen, F.; Duss, A.; Wenzel, L. M.; Buser, C.; Alewell, C. (2018): Soil sealing and unsealing: State of the art and examples. In: Land Degradation and Development 29, 6, 2015-2024. https://doi.org/10.1002/ldr.2919

Tobias, S.; Ströbele, M.; Nobis, M.; Obrist, M.; Moretti, M.; Hunziker, M.; Hersperger, A.; Pütz, M.; Kienast, F.; Buser, T. (2016): Siedlungs- und Landschaftsentwicklung in agglomerationsnahen Räumen. Raumansprüche von Mensch und Natur. Birmensdorf. = Merkblatt für die Praxis 56.

van Beukering, P. J. H.; Brouwer, R.; Koetse, M. J. (2015): Economic values of ecosystem services. In: Bouma, J. A.; van Beukering, P. J. H. (Hrsg.): Ecosystem services. From concept to practice. Cambridge, 89-107. https://doi.org/10.1017/CBO9781107477612

Walz, U. (2013): Landschaftsstrukturmaße und Indikatorensysteme zur Erfassung und Bewertung des Landschafts- 
wandels und seiner Umweltauswirkungen - unter besonderer Berücksichtigung der biologischen Vielfalt. Habilitationsschrift an der Universität Rostock. https://doi.org/ 10.18453/rosdok_id00001159

Weng, Q.; Lu, D. (2008): Mapping urban impervious surfaces from medium and high spatial resolution multispectral imagery. In: Weng, Q. (Hrsg.): Remote Sensing of Impervious Surfaces. Boca Raton, 59-73. https://doi.org/ 10.1201/9781420043754

Wessolek, G. (2014): Bodenüberformung und -versiege- lung. In: Blume, H.-P.; Felix-Henningsen, P.; Frede, H. G.; Guggenberger, G.; Horn, R.; Stahr, K. (Hrsg.): Handbuch der Bodenkunde. Weinheim, 1-33. https://doi. org/10.1002/9783527678495.hbbk2001002

Wiggering, H.; Fischer, J.-U.; Penn-Bressel, G.; Eckelmann, W.; Ekardt, F.; Köpke, U.; Makeschin, F.; Lee, Y. H.; Grimski, D.; Glante, F. (2009): Flächenverbrauch einschränken - jetzt handeln. Empfehlungen der Kommission Bodenschutz beim Umweltbundesamt. Dessau-Roßlau. 